\title{
ERGODIC THEORY FOR QUANTUM SEMIGROUPS
}

\author{
VOLKER RUNDE AND AMI VISELTER
}

\begin{abstract}
Recent results of L. Zsidó, based on his previous work with C. P. Niculescu and A. Ströh, on actions of topological semigroups on von Neumann algebras, give a Jacobs-de Leeuw-Glicksberg splitting theorem at the von Neumann algebra (rather than Hilbert space) level. We generalize this to the framework of actions of quantum semigroups, namely Hopf-von Neumann algebras. To this end, we introduce and study a notion of almost periodic vectors and operators that is suitable for our setting.
\end{abstract}

\section{INTRODUCTION}

The celebrated Jacobs-de Leeuw-Glicksberg splitting theorem [21, 10] is a fundamental result in ergodic theory. It considers a weakly almost periodic semigroup $\mathcal{S}$ of operators over a Banach space $X$, and under an amenability condition, it gives a decomposition of $X$ as the direct sum of the almost periodic vectors of $\mathcal{S}$ and the weakly mixing (or flight) vectors of $\mathcal{S}$. Assume now that $N$ is a von Neumann algebra, $\omega$ is a faithful normal state of $N, G$ is a topological semigroup and $\alpha=\left(\alpha_{s}\right)_{s \in G}$ is an $\omega$-preserving action of $G$ on $N$. The quadruple $(N, \omega, G, \alpha)$ is a noncommutative dynamical system, generalizing the standard ergodic-theoretic setting of a measure-preserving transformation $T$ acting on a probability space $(\Omega, \mu)$, giving rise to the action $f \mapsto f \circ T$, $f \in L^{\infty}(\Omega, \mu)$. On the GNS Hilbert space $\mathcal{H}$ of $(N, \omega)$, one constructs the canonical semigroup $\left(U_{s}\right)_{s \in G}$ of isometries implementing $\alpha$. The Jacobs-de Leeuw-Glicksberg theorem now applies to $\mathcal{S}:=\left\{U_{s}: s \in G\right\}$, yielding a decomposition of $\mathcal{H}$. But in this setting, it is also desirable to obtain a decomposition of $N$ as a direct sum of almost periodic operators and weakly mixing operators. This problem was considered by Niculescu, Ströh and Zsidó [27] in the case $G=\mathbb{Z}_{+}$, and by Zsidó [41] in the general case where $G$ is a locally compact unital semigroup. They define the notion of almost periodicity of an operator in $N$, prove that the set of almost periodic operators forms a von Neumann subalgebra $N^{\mathrm{AP}}$ of $N$, and establish the existence of an $\omega$-preserving conditional expectation from $N$ onto $N^{\mathrm{AP}}$ [27, Theorem 4.2]. The decomposition of $N$ given by this conditional expectation is the desired one.

Extending classical results of ergodic theory to noncommutative dynamical systems has been a central research theme for many years. In addition to the references mentioned above, we refer the reader to Abadie and Dykema [1], Austin, Eisner and Tao [2], Beyers, Duvenhage and Ströh [5], Duvenhage [13, 14, 15], Fidaleo and Mukhamedov [20] and the references therein for (a partial list of) recent works in this spirit. The purpose of the present paper is to extend the results of $[27,41]$ detailed in the previous paragraph to actions of quantum semigroups,

2010 Mathematics Subject Classification. Primary: 20G42, Secondary: 22D25, 37A15, 37A25, 37A30, 46L89.

Key words and phrases. Action, almost periodicity, amenability, Hopf-von Neumann algebra, locally compact quantum group, noncommutative ergodic theorem.

Both authors were supported by NSERC Discovery Grants. 
namely Hopf-von Neumann algebras. In this setting the problems become much more delicate. For instance, it is not obvious at first what the proper definition of almost periodicity should be.

The structure of the paper is as follows. In $\S 1$ we give some background and establish the notation and standing hypothesis. In $\S 2$ we prove a generalized mean ergodic theorem, extending the mean ergodic theorem for Hopf-von Neumann algebras of Duvenhage [13]. This generalization is of independent interest. $\S 3$ is dedicated to (complete) almost periodicity of actions of Hopf-von Neumann algebras: after giving some motivation, we establish the fundamental compactification result (Theorem 3.11) and then give an alternative definition of complete almost periodicity (Corollary 3.14). The main results of the paper are obtained in §4. In particular, we prove that the set $N^{\mathrm{CAP}}$ of completely almost periodic operators is a von Neumann subalgebra of $N$ (Theorem 4.5), and that under certain conditions, there is a canonical conditional expectation from $N$ to $N^{\mathrm{CAP}}$ (Corollary 4.10), which provides the Jacobs-de Leeuw-Glicksberg splitting of $N$.

\section{PRELIMinaries AND NOTATION}

We shall use the notation of Effros and Ruan [16] for operator space terminology. Throughout the paper, the symbols $\odot, \otimes, \otimes_{\min }, \check{\otimes}, \widehat{\otimes}$ and $\bar{\otimes}$ stand for the following respective types of tensor products: the algebraic, Hilbert space, $C^{*}$-algebraic minimal (spatial), operator space injective, projective and normal spatial (including von Neumann algebraic). A projection over a Hilbert space is always orthogonal. In the context of operators over a Hilbert space, "weak" and "strong" refer to the weak and strong operator topologies, respectively. We assume that the reader is familiar with the basics of compact quantum groups (Woronowicz [40], Maes and Van Daele [25]) and the Tomita-Takesaki modular theory [31, 32, 34].

The basic quantum structure we consider in this paper is that of Hopf-von Neumann algebras.

Definition 1.1 ([19]). A Hopf-von Neumann algebra is a pair $\mathbb{G}=\left(L^{\infty}(\mathbb{G}), \Delta\right)$, where $L^{\infty}(\mathbb{G})$ is a von Neumann algebra and $\Delta: L^{\infty}(\mathbb{G}) \rightarrow L^{\infty}(\mathbb{G}) \bar{\otimes} L^{\infty}(\mathbb{G})$ is a co-multiplication, that is, a unital normal $*$-homomorphism which is co-associative: $(\Delta \otimes$ id $) \Delta=($ id $\otimes \Delta) \Delta$.

If $\mathbb{G}$ is a Hopf-von Neumann algebra, we denote $L^{1}(\mathbb{G}):=L^{\infty}(\mathbb{G})_{*}$. This space is a Banach algebra with the product $*$ induced by the predual $\Delta_{*}: L^{1}(\mathbb{G}) \widehat{\otimes} L^{1}(\mathbb{G}) \rightarrow L^{1}(\mathbb{G})$, that is, $\left(\omega_{1} * \omega_{2}\right)(x):=\left(\omega_{1} \otimes \omega_{2}\right) \Delta(x)\left(\omega_{1}, \omega_{2} \in L^{1}(\mathbb{G}), x \in L^{\infty}(\mathbb{G})\right)$.

Definition 1.2. A co-representation of $\mathbb{G}$ is an operator $u \in C \bar{\otimes} L^{\infty}(\mathbb{G})$, for some von Neumann algebra $C$, satisfying

$$
(\mathrm{id} \otimes \Delta)(u)=u_{12} u_{13}
$$

(we use the customary leg numbering notation). If $C=B(\mathcal{K})$ for some Hilbert space $\mathcal{K}$, we say that $u$ is a co-representation of $\mathbb{G}$ on $\mathcal{K}$. A closed subspace $\mathcal{K}^{\prime}$ of $\mathcal{K}$ is said to be invariant under $u$ if $u(p \otimes \mathbb{1})=(p \otimes \mathbb{1}) u(p \otimes \mathbb{1})$, where $p$ is the projection of $\mathcal{K}$ onto $\mathcal{K}^{\prime}$. The restriction $u(p \otimes \mathbb{1}) \in B\left(\mathcal{K}^{\prime}\right) \bar{\otimes} L^{\infty}(\mathbb{G})$ is a typical sub-representation of $\mathbb{G}$.

Definition 1.3. A Hopf-von Neumann algebra $\mathbb{G}$ is called a locally compact quantum group (see Kustermans and Vaes [23, 24] or Van Daele [38], and for an alternative approach see Masuda, 
Nakagami and Woronowicz [26]), abbreviated LCQG, if $L^{\infty}(\mathbb{G})$ admits a pair of normal, semifinite, faithful weights $\varphi, \psi$, called the left and right Haar weights, that are left and right invariant (respectively) in the sense that $\varphi((\omega \otimes$ id $) \Delta(x))=\omega(\mathbb{1}) \varphi(x)$ for all $\omega \in L^{1}(\mathbb{G})^{+}$and $x \in M^{+}$such that $\varphi(x)<\infty$, and $\psi((\operatorname{id} \otimes \omega) \Delta(x))=\omega(\mathbb{1}) \psi(x)$ for all $\omega \in L^{1}(\mathbb{G})^{+}$and $x \in M^{+}$such that $\psi(x)<\infty$.

The deep theory of locally compact quantum groups is not needed in most parts of the paper, especially outside of the appendix.

Definition $1.4([18,11,4])$. Let $\mathbb{G}$ be a Hopf-von Neumann algebra.

(a) We say that $\mathbb{G}$ is amenable if it admits a (two-sided) invariant mean, that is, a state $m \in L^{\infty}(\mathbb{G})^{*}$ with

$$
m((\omega \otimes \mathrm{id}) \Delta(x))=\omega(\mathbb{1}) m(x)=m((\mathrm{id} \otimes \omega) \Delta(x))
$$

for all $x \in L^{\infty}(\mathbb{G}), \omega \in L^{1}(\mathbb{G})$. Recall that if $\mathbb{G}$ is a LCQG, then the existence of either a left- or a right-invariant mean (only one of the equalities in (1.1)) is equivalent to amenability [11, Proposition 3].

(b) We say that $\mathbb{G}$ is (left) co-amenable if $L^{1}(\mathbb{G})$ possesses a bounded left approximate identity.

Remark 1.5. Let $\mathbb{G}$ be an amenable Hopf-von Neumann algebra, and let $m$ be an invariant mean on $\mathbb{G}$. One can use a standard convexity argument (cf. [35, Lemma 3.12, (1)]) to show that there is a net $\left(m_{\kappa}\right)$ of states in $L^{1}(\mathbb{G})$ with $m_{\kappa} \rightarrow m$ in the $\sigma\left(L^{\infty}(\mathbb{G})^{*}, L^{\infty}(\mathbb{G})\right)$-topology and

$$
\lim _{\kappa}\left\|\theta * m_{\kappa}-\theta(\mathbb{1}) m_{\kappa}\right\|=0=\lim _{\kappa}\left\|m_{\kappa} * \theta-\theta(\mathbb{1}) m_{\kappa}\right\| \quad\left(\forall \theta \in L^{1}(\mathbb{G})\right) .
$$

Example 1.6. The two basic examples of LCQGs are the ones that come from a locally compact group $G$ as follows.

(a) Let $L^{\infty}(\mathbb{G})$ be $L^{\infty}(G)$ and define $\Delta$ by $(\Delta(f))(t, s):=f(t s)\left(f \in L^{\infty}(G), s, t \in G\right)$, employing the natural identification $L^{\infty}(G) \bar{\otimes} L^{\infty}(G) \cong L^{\infty}(G \times G)$. Now the product $*$ is the convolution on $L^{1}(\mathbb{G})=L^{1}(G)$. Letting $\varphi, \psi$ be integration against a left and a right Haar measure, respectively, one obtains the commutative $L C Q G \mathbb{G}$ associated with $G$. This $\mathbb{G}$ is always co-amenable, and is amenable if and only if $G$ is amenable as a group.

(b) Let $L^{\infty}(\mathbb{G})$ be $\operatorname{VN}(G)$, the (left) von Neumann algebra of $G$. We define $\Delta$ to be the unique unital normal $*$-homomorphism from $\operatorname{VN}(G)$ to $\operatorname{VN}(G) \bar{\otimes} \mathrm{VN}(G)$ that satisfies $\Delta\left(\lambda_{g}\right)=$ $\lambda_{g} \otimes \lambda_{g}$ for every $g \in G$ ( $\lambda_{g}$ being the left translation by $g$ ), and take both $\varphi, \psi$ to be the Plancherel weight of $G$ [34]. This gives the co-commutative $L C Q G$ associated with $G$. We often write $\hat{G}$ for this $\mathbb{G}$. It is always amenable, and is co-amenable if and only if $G$ is amenable as a group.

Definition 1.7. An action of a Hopf-von Neumann algebra $\mathbb{G}$ on a von Neumann algebra $N$ is a unital normal $*$-homomorphism $\alpha: N \rightarrow N \bar{\otimes} L^{\infty}(\mathbb{G})$ satisfying

$$
(\alpha \otimes \mathrm{id}) \alpha=(\mathrm{id} \otimes \Delta) \alpha .
$$

Standing hypothesis. Throughout the paper we assume that $\mathbb{G}=\left(L^{\infty}(\mathbb{G}), \Delta\right)$ is a Hopf-von Neumann algebra and $N$ is a von Neumann algebra such that the following hold: 
(a) $\mathbb{G}$ is amenable and (left) co-amenable; and

(b) $\alpha$ is an action of $\mathbb{G}$ on $N$, and $\omega$ is a normal faithful state of $N$ invariant under $\alpha$, that is,

$$
(\omega \otimes \mathrm{id}) \alpha=\omega(\cdot) \mathbb{1} .
$$

Furthermore, for some bounded left approximate identity $\left(\epsilon_{\lambda}\right)$ of the Banach algebra $L^{1}(\mathbb{G})$, we have

$$
\left(\mathrm{id} \otimes \epsilon_{\lambda}\right) \alpha(a) \rightarrow a
$$

weakly for every $a \in N$.

Our setting evidently generalizes that of [27] (take $L^{\infty}(\mathbb{G}):=\ell_{\infty}\left(\mathbb{Z}_{+}\right)$and define $\Delta$ as in Example 1.6, (a)).

Remark 1.8. While $N$ should be countably decomposable for $\omega$ to exist, there is no other condition on $N$. We believe it should be possible to obtain stronger results on multiple recurrence in the spirit of Austin, Eisner and Tao [2] if $N$ is assumed finite (also see Remark 4.11).

We let $L^{\infty}(\mathbb{G})$ act on some Hilbert space $L^{2}(\mathbb{G})$ (not necessarily in standard position). We denote by $(\mathcal{H}, \mathrm{id}, \Gamma)$ the GNS construction for $(N, \omega)$. Since $\omega$ is invariant under the action $\alpha$, the isometry $U \in B(\mathcal{H}) \bar{\otimes} L^{\infty}(\mathbb{G})$ determined by

$$
((\operatorname{id} \otimes \theta)(U)) \Gamma(a)=\Gamma((\operatorname{id} \otimes \theta) \alpha(a)) \quad\left(\forall \theta \in L^{1}(\mathbb{G}), a \in N\right)
$$

implements $\alpha$ in the sense that $\alpha(a) U=U(a \otimes \mathbb{1})$ for all $a \in N$. Moreover, $U$ is a co-representation of $\mathbb{G}$.

For every $\zeta \in \mathcal{H}$ we define a bounded operator $T_{\zeta}: L^{1}(\mathbb{G}) \rightarrow \mathcal{H}$ by $T_{\zeta}(\theta):=((\mathrm{id} \otimes \theta)(U)) \zeta$ $\left(\theta \in L^{1}(\mathbb{G})\right)$. We note that Duvenhage [13] denotes $T_{\zeta}(\theta)$ by $\tilde{\theta}^{\alpha} \zeta$.

We shall require a Hilbert space version of $\alpha$ as follows. Denote by $\mathcal{H}_{c}$ the column Hilbert space determined by $\mathcal{H}[16, \S 3.4]$. Since $\Gamma \in \mathcal{C} \mathcal{B}\left(N, \mathcal{H}_{c}\right)$, we have the map $\Gamma \otimes \operatorname{id} \in \mathcal{C} B\left(N \bar{\otimes} L^{\infty}(\mathbb{G}), \mathcal{H}_{c} \bar{\otimes} L^{\infty}(\mathbb{G})\right)$.

Lemma 1.9. The operators $T_{\zeta}, \zeta \in \mathcal{H}$, are completely bounded, and can be regarded as elements of $\mathcal{H}_{c} \bar{\otimes} L^{\infty}(\mathbb{G})$. Furthermore, the operator $\tilde{\alpha}: \mathcal{H}_{c} \rightarrow \mathcal{H}_{c} \bar{\otimes} L^{\infty}(\mathbb{G})$ given by $\tilde{\alpha}(\zeta):=T_{\zeta}$ for all $\zeta \in \mathcal{H}$ is completely contractive. It satisfies $\tilde{\alpha} \circ \Gamma=(\Gamma \otimes \mathrm{id}) \circ \alpha$ and $(\mathrm{id} \otimes \Delta) \tilde{\alpha}=(\tilde{\alpha} \otimes \mathrm{id}) \tilde{\alpha}$ (which belong to $\mathcal{C B}\left(N, \mathcal{H}_{c} \bar{\otimes} L^{\infty}(\mathbb{G})\right)$ and $\mathcal{C B}\left(\mathcal{H}_{c}, \mathcal{H}_{c} \bar{\otimes} L^{\infty}(\mathbb{G}) \bar{\otimes} L^{\infty}(\mathbb{G})\right)$, respectively). Furthermore, (id $\left.\otimes \epsilon_{\lambda}\right) \tilde{\alpha}(\xi) \rightarrow$ $\xi$ weakly for every $\xi \in \mathcal{H}$.

Proof. Recall that the operator space structure of $\mathcal{H}_{c} \bar{\otimes} L^{\infty}(\mathbb{G})$ is given by the natural $w^{*}$-homeomorphic, completely isometric embedding $\mathcal{H}_{c} \bar{\otimes} L^{\infty}(\mathbb{G}) \hookrightarrow \mathcal{C B}\left(L^{1}(\mathbb{G}), \mathcal{H}_{c}\right)$ [16, Theorem 7.2.3 and Corollary 7.1.5]. For every $\zeta \in \mathcal{H}$, the operator $\mathrm{ev}_{\zeta}: B(\mathcal{H}) \rightarrow \mathcal{H}_{c}$ given by $T \mapsto T \zeta, T \in B(\mathcal{H})$, belongs to $\mathcal{C B}\left(B(\mathcal{H}), \mathcal{H}_{c}\right)$ and is $w^{*}$-continuous. Furthermore, the map $\mathcal{H}_{c} \rightarrow \mathcal{C B}\left(B(\mathcal{H}), \mathcal{H}_{c}\right)$ given by $\zeta \mapsto \mathrm{ev}_{\zeta}$ is a complete isometry. Define $\tilde{\alpha}: \mathcal{H}_{c} \rightarrow \mathcal{H}_{c} \bar{\otimes} L^{\infty}(\mathbb{G})$ by $\tilde{\alpha}(\zeta):=\left(\operatorname{ev}_{\zeta} \otimes \mathrm{id}\right)(U)$. From the foregoing and as $\|U\|=1, \tilde{\alpha}$ is completely contractive. By the definition of $T_{\zeta}$, we clearly have $\tilde{\alpha}(\zeta)=T_{\zeta}$ for all $\zeta \in \mathcal{H}$, and from the definition of $U$ we obtain the formula $\tilde{\alpha}(\Gamma(a))=(\Gamma \otimes \mathrm{id}) \alpha(a)$ for all $a \in N$. Consequently, for every $a \in N$,

$$
\begin{aligned}
(\mathrm{id} \otimes \Delta) \tilde{\alpha}(\Gamma(a)) & =(\mathrm{id} \otimes \Delta)(\Gamma \otimes \mathrm{id}) \alpha(a)=(\Gamma \otimes \mathrm{id} \otimes \mathrm{id})(\mathrm{id} \otimes \Delta) \alpha(a) \\
& =(\Gamma \otimes \mathrm{id} \otimes \mathrm{id})(\alpha \otimes \mathrm{id}) \alpha(a)=(\tilde{\alpha} \otimes \mathrm{id})(\Gamma \otimes \mathrm{id}) \alpha(a)=(\tilde{\alpha} \otimes \mathrm{id}) \tilde{\alpha}(\Gamma(a)) .
\end{aligned}
$$


Hence $(\mathrm{id} \otimes \Delta) \tilde{\alpha}=(\tilde{\alpha} \otimes \mathrm{id}) \tilde{\alpha}$. By assumption, $\left(\mathrm{id} \otimes \epsilon_{\lambda}\right) \alpha(a) \rightarrow a$ weakly for all $a \in N$, so that

$$
\left(\mathrm{id} \otimes \epsilon_{\lambda}\right) \tilde{\alpha}(\Gamma(a))=\Gamma\left(\left(\mathrm{id} \otimes \epsilon_{\lambda}\right) \alpha(a)\right) \rightarrow \Gamma(a)
$$

weakly. From the boundedness of $\left(\epsilon_{\lambda}\right)$ we infer that $\left(\mathrm{id} \otimes \epsilon_{\lambda}\right) \tilde{\alpha}(\xi) \rightarrow \xi$ weakly for every $\xi \in \mathcal{H}$. This completes the proof.

Remark 1.10. For $\zeta \in \mathcal{H}$ and $\theta, \vartheta \in L^{1}(\mathbb{G})$, we have

$$
T_{T_{\zeta}(\vartheta)}=\tilde{\alpha}((\mathrm{id} \otimes \vartheta) \tilde{\alpha}(\zeta))=(\mathrm{id} \otimes \mathrm{id} \otimes \vartheta)(\tilde{\alpha} \otimes \mathrm{id}) \tilde{\alpha}(\zeta)=(\mathrm{id} \otimes \mathrm{id} \otimes \vartheta)(\mathrm{id} \otimes \Delta) \tilde{\alpha}(\zeta)
$$

Thus

$$
T_{T_{\zeta}(\vartheta)}(\theta)=(\mathrm{id} \otimes \theta \otimes \vartheta)(\mathrm{id} \otimes \Delta) \tilde{\alpha}(\zeta)=(\mathrm{id} \otimes(\theta * \vartheta)) \tilde{\alpha}(\zeta)=T_{\zeta}(\theta * \vartheta) .
$$

For the definition of a compact quantum group we use the $C^{*}$-algebraic language, which is more suitable for our purposes, although there is an equivalent von Neumann algebraic one.

Definition 1.11 ([40, 25]). A compact quantum group is a pair $\mathbb{H}=(C(\mathbb{H}), \Delta)$, where $C(\mathbb{H})$ is a unital $C^{*}$-algebra, $\Delta: C(\mathbb{H}) \rightarrow C(\mathbb{H}) \otimes_{\min } C(\mathbb{H})$ is a $C^{*}$-algebraic co-multiplication, that is, a unital $*$-homomorphism which is co-associative, i.e., $(\Delta \otimes \mathrm{id}) \Delta=(\mathrm{id} \otimes \Delta) \Delta$, and furthermore, the sets $(C(\mathbb{H}) \otimes \mathbb{1}) \Delta(C(\mathbb{H}))$ and $(\mathbb{1} \otimes C(\mathbb{H})) \Delta(C(\mathbb{H}))$ are norm total in $C(\mathbb{H}) \otimes_{\min } C(\mathbb{H})$.

If the pair $(C(\mathbb{H}), \Delta)$ satisfies all these assumptions apart from the density conditions, it is called a compact quantum semigroup.

\section{BASIC ERGODIC THEORY}

Lemma 2.1. Let $C$ be a von Neumann algebra and $u \in C \bar{\otimes} L^{\infty}(\mathbb{G})$. Fix $\mu \in C_{*}^{+}$, and denote its GNS construction by $\left(\mathcal{H}_{\mu}, \mathrm{id}, \Gamma_{\mu}\right)$. Denote $\Xi:=\Gamma_{\mu} \otimes \Gamma$. For every $\theta \in L^{1}(\mathbb{G})^{+}$, the operator $V_{u}(\theta)$ given by

$$
V_{u}(\theta): \Xi(a) \mapsto \Xi\left[(\mathrm{id} \otimes \mathrm{id} \otimes \theta)\left(u_{13} \cdot(\mathrm{id} \otimes \alpha)(a)\right)\right] \quad(\forall a \in C \bar{\otimes} N)
$$

extends to an element of $B\left(\mathcal{H}_{\mu} \otimes \mathcal{H}\right)$ satisfying $\left\|V_{u}(\theta)\right\| \leq\|u\|\|\theta\|$. Moreover, if $u$ is a co-representation of $\mathbb{G}$, then $V_{u}\left(\theta_{1}\right) V_{u}\left(\theta_{2}\right)=V_{u}\left(\theta_{1} * \theta_{2}\right)$ for all $\theta_{1}, \theta_{2} \in L^{1}(\mathbb{G})^{+}$.

Proof. Let $a, b \in C \bar{\otimes} N$. Since $\alpha$ is $\omega$-invariant, we have

$$
\begin{aligned}
\mid\left\langle V_{u}(\theta) \Xi(a),\right. & \Xi(b)\rangle \mid \\
& =\left|(\mu \otimes \omega)\left[b^{*} \cdot(\mathrm{id} \otimes \mathrm{id} \otimes \theta)\left(u_{13}(\mathrm{id} \otimes \alpha)(a)\right)\right]\right|=\left|(\mu \otimes \omega \otimes \theta)\left(b_{12}^{*} u_{13}(\mathrm{id} \otimes \alpha)(a)\right)\right| \\
& \leq\|\theta\|^{1 / 2}\|\Xi(b)\|(\mu \otimes \omega \otimes \theta)\left((\mathrm{id} \otimes \alpha)\left(a^{*}\right) u_{13}^{*} u_{13}(\mathrm{id} \otimes \alpha)(a)\right)^{1 / 2} \\
& \leq\|\theta\|^{1 / 2}\|\Xi(b)\|\|u\|(\mu \otimes \omega \otimes \theta)\left((\mathrm{id} \otimes \alpha)\left(a^{*} a\right)\right)^{1 / 2}=\|\theta\|\|\Xi(b)\|\|\Xi(a)\|\|u\|
\end{aligned}
$$

by the Cauchy-Schwarz inequality. This proves the first assertion. If $u$ is a co-representation of $\mathbb{G}, \theta_{1}, \theta_{2} \in L^{1}(\mathbb{G})^{+}$and $a \in C \bar{\otimes} N$, then

$$
\begin{aligned}
V_{u}\left(\theta_{1}\right) V_{u}\left(\theta_{2}\right) \Xi(a) & =V_{u}\left(\theta_{1}\right) \Xi\left[\left(\mathrm{id} \otimes \mathrm{id} \otimes \theta_{2}\right)\left(u_{13} \cdot(\mathrm{id} \otimes \alpha)(a)\right)\right] \\
& =\Xi\left\{\left(\mathrm{id} \otimes \mathrm{id} \otimes \theta_{1}\right)\left[u_{13} \cdot(\mathrm{id} \otimes \alpha)\left(\mathrm{id} \otimes \mathrm{id} \otimes \theta_{2}\right)\left(u_{13} \cdot(\mathrm{id} \otimes \alpha)(a)\right)\right]\right\} \\
& =\Xi\left\{\left(\mathrm{id} \otimes \mathrm{id} \otimes \theta_{1} \otimes \theta_{2}\right)\left[u_{13} u_{14} \cdot(\mathrm{id} \otimes(\alpha \otimes \mathrm{id}) \alpha)(a)\right]\right\} .
\end{aligned}
$$


Using that $\alpha$ is an action and $u$ is a co-representation, we get

$$
\begin{aligned}
V_{u}\left(\theta_{1}\right) V_{u}\left(\theta_{2}\right) \Xi(a) & =\Xi\left\{\left(\mathrm{id} \otimes \mathrm{id} \otimes \theta_{1} \otimes \theta_{2}\right)\left[(\mathrm{id} \otimes \mathrm{id} \otimes \Delta)\left(u_{13}(\mathrm{id} \otimes \alpha)(a)\right)\right]\right\} \\
& =\Xi\left[\left(\mathrm{id} \otimes \mathrm{id} \otimes\left(\theta_{1} * \theta_{2}\right)\right)\left(u_{13}(\mathrm{id} \otimes \alpha)(a)\right)\right]=V_{u}\left(\theta_{1} * \theta_{2}\right) \Xi(a) .
\end{aligned}
$$

Hence $V_{u}\left(\theta_{1}\right) V_{u}\left(\theta_{2}\right)=V_{u}\left(\theta_{1} * \theta_{2}\right)$ by continuity.

The next result is a generalized mean ergodic theorem (cf. [27, Proposition 3.2]) for Hopf-von Neumann algebras. When taking $u$ to be the trivial representation, one recovers Duvenhage's result [13].

Theorem 2.2. Let $C$ be a von Neumann algebra and $u \in C \bar{\otimes} L^{\infty}(\mathbb{G})$ be a unitary co-representation of $\mathbb{G}$. Fix $\mu \in C_{*}^{+}$, denote its GNS construction by $\left(\mathcal{H}_{\mu}, \mathrm{id}, \Gamma_{\mu}\right)$ and let $\Xi:=\Gamma_{\mu} \otimes \Gamma$. Denote by $P_{u}$ the projection (over $\mathcal{H}_{\mu} \otimes \mathcal{H}$ ) onto the space

$$
\bigcap_{\theta \text { is a state in } L^{1}(\mathbb{G})} \operatorname{ker}\left(V_{u}(\theta)-\mathbb{1}\right) .
$$

Let $\left(m_{\kappa}\right)$ be a net of states in $L^{1}(\mathbb{G})$ such that $\left\|m_{\kappa} * \theta-m_{\kappa}\right\| \rightarrow 0$ and $\theta * m_{\kappa}-m_{\kappa} \rightarrow 0$ in the $\sigma\left(L^{1}(\mathbb{G}), L^{\infty}(\mathbb{G})\right)$-topology for each state $\theta$ in $L^{1}(\mathbb{G})$ (see Remark 1.5).

(a) We have $V_{u}\left(m_{\kappa}\right) \rightarrow P_{u}$ strongly.

(b) Suppose that $\mu$ is faithful. There exists a unique projection $E_{u}$ from $C \bar{\otimes} N$ onto the subspace $N_{u}:=\left\{b \in C \bar{\otimes} N:(\mathrm{id} \otimes \alpha)(b)=u_{13}^{*} b_{12}\right\}$ satisfying $\Xi \circ E_{u}=P_{u} \circ \Xi$. Furthermore, $E_{u}$ is normal, it has norm 1 (unless $E_{u}=0$ ),

$$
\left(\mathrm{id} \otimes \mathrm{id} \otimes m_{\kappa}\right)\left[u_{13} \cdot(\mathrm{id} \otimes \alpha)(a)\right] \rightarrow E_{u}(a)
$$

strongly for every $a \in C \bar{\otimes} N$ and $\left(m_{\kappa}\right)$ as above and $\overline{\Xi\left(N_{u}\right)}=\operatorname{Im} P_{u}$.

Remark 2.3. We emphasize that neither $P_{u}$ nor $E_{u}$ depend on the chosen net $\left(m_{\kappa}\right)$.

Proof of Theorem 2.2. (a) Recall that for any contraction $v \in B(\mathcal{H})$ we have $\operatorname{ker}(v-\mathbb{1})=\operatorname{ker}\left(v^{*}-\right.$ $\mathbb{1})$, thus $\operatorname{ker}(v-\mathbb{1})^{\perp}=\overline{\operatorname{Im}(v-\mathbb{1})}$. Therefore $\operatorname{Im}\left(\mathbb{1}-P_{u}\right)=\overline{\operatorname{span}} \bigcup_{\theta} \operatorname{Im}\left(V_{u}(\theta)-\mathbb{1}\right)$, where the union goes over all states in $L^{1}(\mathbb{G})$. For every state $\theta \in L^{1}(\mathbb{G})$, we have $V_{u}\left(m_{\kappa}\right)\left(V_{u}(\theta)-\mathbb{1}\right)=$ $V_{u}\left(m_{\kappa} * \theta-m_{\kappa}\right)$, and hence $\left\|V_{u}\left(m_{\kappa}\right)\left(V_{u}(\theta)-\mathbb{1}\right)\right\| \leq\left\|m_{\kappa} * \theta-m_{\kappa}\right\| \rightarrow 0$. Thus $V_{u}\left(m_{\kappa}\right) \eta \rightarrow 0$ for every $\eta$ in the dense subspace $\operatorname{span} \bigcup_{\theta} \operatorname{Im}\left(V_{u}(\theta)-\mathbb{1}\right)$ of $\operatorname{Im}\left(\mathbb{1}-P_{u}\right)$. Since $\left(V_{u}\left(m_{\kappa}\right)\right)_{\kappa}$ is uniformly bounded by 1 , we deduce that $V_{u}\left(m_{\kappa}\right) \eta \rightarrow 0$ for all $\eta \in \operatorname{Im}\left(\mathbb{1}-P_{u}\right)$. Now, for all $\zeta \in \mathcal{H}{ }_{\mu} \otimes \mathcal{H}$, we have

$$
V_{u}\left(m_{\kappa}\right) \zeta=V_{u}\left(m_{\kappa}\right) P_{u} \zeta+V_{u}\left(m_{\kappa}\right)\left(\mathbb{1}-P_{u}\right) \zeta=P_{u} \zeta+V_{u}\left(m_{\kappa}\right)\left(\mathbb{1}-P_{u}\right) \zeta \rightarrow P_{u} \zeta .
$$

(b) Let $a \in C \bar{\otimes} N$ be given. The net $\left(\left(\mathrm{id} \otimes \mathrm{id} \otimes m_{\kappa}\right)\left(u_{13} \cdot(\mathrm{id} \otimes \alpha)(a)\right)\right)_{\lambda}$ is bounded by $\|a\|$, so it admits a weak cluster point in $C \bar{\otimes} N$, denoted $E_{u}(a)$, with $\left\|E_{u}(a)\right\| \leq\|a\|$. By (a) we have

$$
\Xi\left[\left(\mathrm{id} \otimes \mathrm{id} \otimes m_{\kappa}\right)\left(u_{13} \cdot(\mathrm{id} \otimes \alpha)(a)\right)\right]=V_{u}\left(m_{\kappa}\right) \Xi(a) \rightarrow P_{u} \Xi(a),
$$

from which the identity $\Xi\left(E_{u}(a)\right)=P_{u} \Xi(a)$ is obtained using (a). This proves that $E_{u}$ does not depend on the particularly chosen net $\left(m_{\kappa}\right)$ and that it is normal. Moreover, (2.1) entails that 
actually $\left(\mathrm{id} \otimes \mathrm{id} \otimes m_{\kappa}\right)\left(u_{13} \cdot(\mathrm{id} \otimes \alpha)(a)\right) \rightarrow E_{u}(a)$ strongly, as we have convergence at the vector $\Xi(\mathbb{1})$, which is separating for $C \bar{\otimes} N$ on $\mathcal{H}_{\mu} \otimes \mathcal{H}$.

We now prove that $E_{u}(a) \in N_{u}$. As $\alpha$ is an action, we have

$$
\begin{aligned}
(\mathrm{id} \otimes \alpha)\left(E_{u}(a)\right) & =\lim _{\kappa}\left(\mathrm{id} \otimes \mathrm{id} \otimes \mathrm{id} \otimes m_{\kappa}\right)(\mathrm{id} \otimes \alpha \otimes \mathrm{id})\left[u_{13} \cdot(\mathrm{id} \otimes \alpha)(a)\right] \\
& =\lim _{\kappa}\left(\mathrm{id} \otimes \mathrm{id} \otimes \mathrm{id} \otimes m_{\kappa}\right)\left[u_{14} \cdot(\mathrm{id} \otimes(\alpha \otimes \mathrm{id}) \alpha)(a)\right] \\
& =\lim _{\kappa}\left(\mathrm{id} \otimes \mathrm{id} \otimes \mathrm{id} \otimes m_{\kappa}\right)\left[u_{14} \cdot(\mathrm{id} \otimes(\mathrm{id} \otimes \Delta) \alpha)(a)\right]
\end{aligned}
$$

(all limits are weak ones). Thus, if $\theta \in L^{1}(\mathbb{G})$ is a state, then by approximate invariance of $\left(m_{\kappa}\right)$,

$$
\begin{aligned}
(\mathrm{id} \otimes \mathrm{id} \otimes \theta)\left[u_{13} \cdot(\mathrm{id} \otimes \alpha)\left(E_{u}(a)\right)\right] & =\lim _{\kappa}\left(\mathrm{id} \otimes \mathrm{id} \otimes \theta \otimes m_{\kappa}\right)\left[u_{13} u_{14} \cdot(\mathrm{id} \otimes(\mathrm{id} \otimes \Delta) \alpha)(a)\right] \\
& =\lim _{\kappa}\left(\mathrm{id} \otimes \mathrm{id} \otimes \theta \otimes m_{\kappa}\right)(\mathrm{id} \otimes \mathrm{id} \otimes \Delta)\left[u_{13}(\mathrm{id} \otimes \alpha)(a)\right] \\
& =\lim _{\kappa}\left(\mathrm{id} \otimes \mathrm{id} \otimes\left(\theta * m_{\kappa}\right)\right)\left[u_{13}(\mathrm{id} \otimes \alpha)(a)\right] \\
& =\lim _{\kappa}\left(\mathrm{id} \otimes \mathrm{id} \otimes m_{\kappa}\right)\left[u_{13}(\mathrm{id} \otimes \alpha)(a)\right]=E_{u}(a) .
\end{aligned}
$$

Hence $u_{13} \cdot(\mathrm{id} \otimes \alpha)\left(E_{u}(a)\right)=E_{u}(a) \otimes \mathbb{1}$, and $u$ being a unitary, we obtain $E_{u}(a) \in N_{u}$. On the other hand, we obviously have $E_{u}(a)=a$ if $a \in N_{u}$. We conclude that $E_{u}$ is a projection from $C \bar{\otimes} N$ onto $N_{u}$.

The inclusion $\Xi\left(N_{u}\right) \subseteq \operatorname{Im} P_{u}$ is clear. To prove that $\operatorname{Im} P_{u} \subseteq \overline{\Xi\left(N_{u}\right)}$, let $\zeta \in \operatorname{Im} P_{u}$ and $\varepsilon>0$ be given. Pick $a \in C \bar{\otimes} N$ with $\|\zeta-\Xi(a)\| \leq \varepsilon$. Then $\zeta-\Xi\left(E_{u}(a)\right)=P_{u}(\zeta-\Xi(a))$ has norm $\leq \varepsilon$ and $E_{u}(a) \in N_{u}$.

\section{COMplete Almost PERIOdicity}

The aim of this section is to give a definition of almost periodic vectors and operators that is adequate for our setting of actions of Hopf-von Neumann algebras, and then generalize the compactification result [27, Lemma 4.1]. This is an essential step for the rest of the paper.

The question of how to define almost periodicity in the quantum setting is a nontrivial one. We begin by recalling the classical setting of Niculescu, Ströh and Zsidó [27] and Zsidó [41]. Let $N$ be a von Neumann algebra, $\omega \in N_{*}$ a faithful state, $G$ a locally compact unital semigroup, and $\alpha=\left(\alpha_{s}\right)_{s \in G}$ a (weakly continuous) action of $G$ on $N$. We denote the GNS construction for $(N, \omega)$ by $(\mathcal{H}, \Gamma)$. For $s \in G$, the isometry $U_{s} \in B(\mathcal{H})$ determined by $U_{s} \Gamma(a):=\Gamma\left(\alpha_{s}(a)\right)$, $a \in N$, implements $\alpha_{s}$ in the sense that $\alpha_{s}(a) U_{s}=U_{s} a$ for all $a \in N$. Moreover, $U=\left(U_{s}\right)_{s \in G}$ is a representation of $G: U_{s} U_{t}=U_{s t}$ for every $s, t \in G$. In $[27,41]$, a vector $\zeta \in \mathcal{H}$ is called almost periodic if its orbit $\left\{U_{s} \zeta: s \in G\right\}$ is relatively compact in $\mathcal{H}$. In this setting, however, there are other notions of almost periodicity $[21,10]$. We summarize the approaches to defining the set of almost periodic vectors:

(i) the closed linear span of the unitary subspaces (Jacobs [21], de Leeuw and Glicksberg [10, Definition of $B_{p}$, p. 75]);

(ii) the reversible vectors (Jacobs [21], de Leeuw and Glicksberg [10, Definition of $B_{r}$, p. 73]);

(iii) the vectors whose orbits are relatively compact [27, 41] (see above). 
Approaches (i) and (ii) are equivalent by [10, Theorem 4.10], while their equivalence to (iii) is a consequence of a result similar to [27, Lemma 4.1] together with the theory of unitary representations of compact groups. Approaches (ii) and (iii), which rely on the period of the vector, have the clear advantage of being far more tangible than (i) when testing for almost periodicity.

In the quantum setting, it is desirable to find a definition that would be an obvious generalization of (iii). Nevertheless, it seems that the only feasible definition in general is a standard adaptation of (i). In Subsection 3.1 we give some motivation to almost periodicity in the quantum setting. It is not essential for understanding the rest of the paper, but it does put things in the right perspective. In Subsection 3.2 we introduce our definition (3.7) of complete almost periodicity and prove the fundamental compactification result, Theorem 3.11. Then we show in Corollary 3.14 that under some assumptions, it is indeed possible to give an equivalent definition of complete almost periodicity, which is very close to (iii).

3.1. Motivation. The following easy lemma serves as some motivation for a possible generalization of almost periodicity to the quantum setting.

Lemma 3.1. In the classical setting of [27, 41] (see the introduction to this section), suppose that $\mu$ is a positive Borel measure on $G$ that is finite on compact sets and nonzero on open sets. For $\zeta \in \mathcal{H}$, define $T_{\zeta} \in B\left(L^{1}(G, \mu), \mathcal{H}\right)$ by $T_{\zeta}(\theta):=\int_{G} \theta(s) U_{s} \zeta \mathrm{d} s, \theta \in L^{1}(G, \mu)$. Then $\zeta$ is almost periodic $\Longleftrightarrow T_{\zeta}$ is compact.

Proof. Let $\zeta \in \mathcal{H}$. By Mazur's theorem [12, Theorem V.2.6], $\zeta$ is almost periodic if and only if $\overline{\mathrm{co}}\left\{U_{s} \zeta: s \in G\right\}$ is compact. For every $\theta \in L^{1}(G)$ with $\theta \geq 0$ and $\|\theta\|_{1}=1$, we have $T_{\zeta}(\theta) \in$ $\overline{\mathrm{co}}\left\{U_{s} \zeta: s \in G\right\}$. Moreover, $U_{s} \zeta \in \overline{\left\{T_{\zeta}(\theta): 0 \leq \theta \in L^{1}(G),\|\theta\|_{1}=1\right\}}$ for all $s \in G$. Hence the assertion follows.

As normally happens when one moves from the classical to the quantum setting, Banach space ("commutative") notions are replaced by their operator space ("noncommutative") counterparts.

Definition 3.2 ([29]). Let $E, F$ be operator spaces and $T \in \mathcal{C B}(E, F)$. We say that $T$ is completely compact if for every $\varepsilon>0$ there exists a finite-dimensional subspace $F_{\varepsilon}$ of $F$ such that $\left\|Q_{F_{\varepsilon}} T\right\|_{\mathrm{cb}}<$ $\varepsilon$, where $Q_{F_{\varepsilon}}: F \rightarrow F / F_{\varepsilon}$ is the quotient map. The space of these operators is denoted by $\mathcal{C K}(E, F)$.

Complete compactness implies compactness, and the two notions agree when $E$ has the maximal operator space structure (e.g., in the classical setting). The space $\mathcal{C K}(E, F)$ contains the cb-closure of the finite-rank operators from $E$ to $F$, that is, the space of all operators in $\mathcal{C B}(E, F)$ that may be viewed as elements of the injective tensor product $E^{*} \check{\otimes} F$ via its completely isometric embedding in $\mathcal{C B}(E, F)$ [16, Proposition 8.1.2]. These two spaces actually coincide when $F$ is a dual operator space and $E^{*}, F$ are injective [29, Proposition 1.6]. Returning to our setting, we consider the operators $T_{\zeta} \in \mathcal{C B}\left(L^{1}(\mathbb{G}), \mathcal{H}_{c}\right), \zeta \in \mathcal{H}$ (see $\S 1$ ). If $\mathbb{G}$ is a LCQG, then co-amenability implies that $L^{\infty}(\mathbb{G})=L^{1}(\mathbb{G})^{*}$ is injective [4]. All the foregoing suggests that the quantum version of almost periodicity of a vector $\zeta \in \mathcal{H}$ should be based on $\tilde{\alpha}(\zeta)=T_{\zeta}$ belonging to $\mathcal{H}_{c} \check{\otimes} L^{\infty}(\mathbb{G})$ $\left(\hookrightarrow \mathcal{H}_{c} \bar{\otimes} L^{\infty}(\mathbb{G}) \hookrightarrow \mathcal{C B}\left(L^{1}(\mathbb{G}), \mathcal{H}_{c}\right)\right)$. 
Lemma 3.3. Set $\mathcal{K}:=\left\{\zeta \in \mathcal{H}: \tilde{\alpha}(\zeta) \in \mathcal{H}_{c} \check{\otimes} L^{\infty}(\mathbb{G})\right\}$. Then $\mathcal{K}$ is a closed subspace of $\mathcal{H}$, and we have $\tilde{\alpha}(\mathcal{K}) \subseteq \mathcal{K}_{c} \check{\otimes} L^{\infty}(\mathbb{G})$ and $\tilde{\alpha}\left(\overline{\Gamma \Gamma^{-1}(\mathcal{K})}\right) \subseteq \overline{\Gamma \Gamma^{-1}(\mathcal{K})}{ }_{c} \check{\otimes} L^{\infty}(\mathbb{G})$.

Proof. That $\mathcal{K}$ is a closed subspace of $\mathcal{H}$ follows easily from Lemma 1.9. To show that $\tilde{\alpha}(\mathcal{K}) \subseteq$ $\mathcal{K}_{c} \check{\otimes} L^{\infty}(\mathbb{G})$, let $\zeta \in \mathcal{K}$. Since $\tilde{\alpha}(\zeta)=T_{\zeta} \in \mathcal{H}_{c} \check{\otimes} L^{\infty}(\mathbb{G})$, we need to prove that $\operatorname{Im} T_{\zeta} \subseteq \mathcal{K}$; indeed, this is enough because of the existence of the (orthogonal, thus completely contractive) projection from $\mathcal{H}$ onto $\mathcal{K}$. Fix $\vartheta \in L^{1}(\mathbb{G})$. For every $\theta \in L^{1}(\mathbb{G})$ we have $T_{T_{\zeta}(\vartheta)}(\theta)=T_{\zeta}(\theta * \vartheta)$ (Remark 1.10), so letting $S: L^{1}(\mathbb{G}) \rightarrow L^{1}(\mathbb{G})$ be given by $\theta \mapsto \theta * \vartheta$, we conclude that $T_{T_{\zeta}(\vartheta)}=$ $T_{\zeta} S$. Since $T_{\zeta} \in \mathcal{H}_{c} \check{\otimes} L^{\infty}(\mathbb{G})$ and $S \in \mathcal{C B}\left(L^{1}(\mathbb{G})\right)$, we have $T_{T_{\zeta}(\vartheta)} \in \mathcal{H}_{c} \check{\otimes} L^{\infty}(\mathbb{G})$, i.e., $T_{\zeta}(\vartheta) \in \mathcal{K}$. The last assertion follows similarly as $T_{\Gamma(a)}(\theta)=\Gamma((\mathrm{id} \otimes \theta) \alpha(a))$ for all $a \in N$ and $\theta \in L^{1}(\mathbb{G})$.

Corollary 3.4. For every $a \in \Gamma^{-1}(\mathcal{K})$ we have $\alpha(a) \in \Gamma^{-1}(\mathcal{K}) \bar{\otimes}_{\mathcal{F}} L^{\infty}(\mathbb{G})$, where $\bar{\otimes}_{\mathcal{F}}$ stands for the normal Fubini tensor product of dual operator spaces.

Lemma 3.5. Let $E$ be an operator space and $\mathcal{K}$ be a Hilbert space. Let $x \in \mathcal{K}_{c} \check{\otimes} E$, and define $E_{x}:=\overline{\operatorname{span}}\left\{\left(\rho^{*} \otimes \mathrm{id}\right) x: \rho \in \mathcal{K}\right\}$. Then $x \in \mathcal{K}_{c} \check{\otimes} E_{x}$.

The elementary proof is left to the reader.

Proposition 3.6. Set again $\mathcal{K}:=\left\{\zeta \in \mathcal{H}: \tilde{\alpha}(\zeta) \in \mathcal{H}_{c} \check{\otimes} L^{\infty}(\mathbb{G})\right\}$, and let $\mathcal{A}$ be the unital $C^{*}$ subalgebra of $L^{\infty}(\mathbb{G})$ generated by $\left\{\left(\eta^{*} \otimes \mathrm{id}\right) \tilde{\alpha}(\zeta): \zeta \in \mathcal{K}, \eta \in \mathcal{H}\right\}$. Then $\Delta$ restricts to a $C^{*}$-algebraic co-multiplication $\mathcal{A} \rightarrow \mathcal{A} \otimes_{\min } \mathcal{A}$. In the case that $\mathbb{G}$ is a $L C Q G$, $\mathcal{A}$ is a $C^{*}$-subalgebra of $M\left(C_{0}(\mathbb{G})\right)$.

Proof. It follows from Lemmas 3.3 and 3.5 that $\tilde{\alpha}(\mathcal{K}) \subseteq \mathcal{K}_{c} \check{\otimes} \mathcal{A}$. Using the formula (id $\left.\otimes \Delta\right) \tilde{\alpha}=$ $(\tilde{\alpha} \otimes \mathrm{id}) \tilde{\alpha}$ of Lemma 1.9 we have for $\zeta \in \mathcal{K}, \eta \in \mathcal{H}$,

$$
\Delta\left(\left(\eta^{*} \otimes \mathrm{id}\right) \tilde{\alpha}(\zeta)\right)=\left(\eta^{*} \otimes \mathrm{id} \otimes \mathrm{id}\right)(\mathrm{id} \otimes \Delta) \tilde{\alpha}(\zeta)=\left(\eta^{*} \otimes \mathrm{id} \otimes \mathrm{id}\right)(\tilde{\alpha} \otimes \mathrm{id}) \tilde{\alpha}(\zeta) \in \mathcal{A} \otimes_{\min } \mathcal{A},
$$

since for $C^{*}$-algebras, the minimal and the operator space injective tensor products coincide. Consequently, $\Delta(\mathcal{A}) \subseteq \mathcal{A} \otimes_{\min } \mathcal{A}$. In the case that $\mathbb{G}$ is a LCQG, we have $U \in M\left(\mathbb{K}(\mathcal{H}) \otimes_{\min } C_{0}(\mathbb{G})\right)$ because $U$ is a co-representation of $\mathbb{G}$ (this is folklore; see, e.g., [7], Corollary 4.12 and the proof of Theorem 4.9), and since $\left(\eta^{*} \otimes \mathrm{id}\right) \tilde{\alpha}(\zeta)=\left(\omega_{\zeta, \eta} \otimes \mathrm{id}\right)(U)$ for all $\zeta, \eta \in \mathcal{H}$, we obtain that $\mathcal{A} \subseteq M\left(C_{0}(\mathbb{G})\right)$.

The pair $\left(\mathcal{A},\left.\Delta\right|_{\mathcal{A}}\right)$ is, therefore, a compact quantum semigroup (which is a compactification of $\mathbb{G})$, but normally not a compact quantum group.

3.2. The compactification. The previous discussion indicates that optimally, we would say that $\zeta \in \mathcal{H}$ is almost periodic in the quantum setting if $\tilde{\alpha}(\zeta) \in \mathcal{H}_{c} \check{\otimes} L^{\infty}(\mathbb{G})$. Nonetheless, this definition is too weak for the development of the rest of the theory, and we now introduce a more restrictive one, as follows. It is the noncommutative version of approach (i) above (also compare Sołtan [30] and Woronowicz [39]).

Definition 3.7. The set $\mathcal{H}^{\mathrm{CP}}$ of completely periodic vectors consists of all $\zeta \in \mathcal{H}$ with the following property: there exists a finite-dimensional sub-representation $u$ of $U$ on a space that contains $\zeta$, such that both $u$ and $u^{\mathrm{t}}$ are invertible. ${ }^{1}$

\footnotetext{
${ }^{1}$ Here $u^{\mathrm{t}}$ is the transpose of $u$, with respect to some basis of the space.
} 
The completely almost periodic vectors are the elements of $\mathcal{H}^{\mathrm{CAP}}:=\overline{\mathrm{span}} \mathcal{H}^{\mathrm{CP}}$, and the completely almost periodic operators are the elements of $N^{\mathrm{CAP}}:=\Gamma^{-1}\left(\mathcal{H}^{\mathrm{CAP}}\right)$.

We will give in Corollary 3.14 another characterization of $\mathcal{H}^{\mathrm{CAP}}$ under additional assumptions. Remark 3.8. The set $N^{\mathrm{CAP}}$ is a weakly closed subspace of $N$.

The main objective of this paper is to address the following questions:

(A) Is $N^{\mathrm{CAP}}$ a von Neumann algebra?

(B) Is $\Gamma\left(N^{\mathrm{CAP}}\right)$ dense in $\mathcal{H}^{\mathrm{CAP}}$ ?

(C) Is $N^{\text {CAP }}$ globally invariant under the modular automorphism group $\sigma^{\omega}$ of $\omega$ ?

Definition 3.9. Let $\mathbb{G}$ be a Hopf-von Neumann algebra. A compact quantum group $\mathbb{H}=$ $\left(C(\mathbb{H}), \Delta_{\mathbb{H}}\right)$ is a compactification of $\mathbb{G}$ if $C(\mathbb{H}) \subseteq L^{\infty}(\mathbb{G})$ and $\Delta_{\mathbb{H}}=\left.\Delta\right|_{C(\mathbb{H})}$. If $\mathbb{G}$ is a LCQG, we further require that $C(\mathbb{H}) \subseteq M\left(C_{0}(\mathbb{G})\right)$.

Remark 3.10. If $\mathbb{G}$ is co-amenable, then so is $\mathbb{H}$, because if $\left(\epsilon_{\lambda}\right)$ is a bounded left approximate identity of $\mathbb{G}$, then any cluster point of $\left(\left.\epsilon_{\lambda}\right|_{C(\mathbb{H})}\right)$ in $C(\mathbb{H})^{*}$ is a co-unit of $\mathbb{H}$ (cf. Bédos and Tuset [4, Theorem 3.1]).

Theorem 3.11. There exists a co-amenable compactification $\mathbb{H}=\left(C(\mathbb{H}), \Delta_{\mathbb{H}}\right)$ of $\mathbb{G}$ such that $\left.U\right|_{\mathcal{H}^{C A P} \otimes L^{2}(\mathbb{G})}$ is a unitary co-representation of $\mathbb{H}$ on $\mathcal{H}^{\mathrm{CAP}}$ in the $C^{*}$-algebraic sense. To elaborate, $C(\mathbb{H})$ is the unital $C^{*}$-subalgebra of $L^{\infty}(\mathbb{G})$ generated by $\left\{\left(\omega_{\zeta, \eta} \otimes \mathrm{id}\right)(U): \zeta \in \mathcal{H}^{\mathrm{CAP}}, \eta \in \mathcal{H}\right\}$ and $\Delta_{\mathbb{H}}$ is the restriction of $\Delta$ to $C(\mathbb{H})$.

Proof. From Definition 3.7 it follows that $\Delta(C(\mathbb{H})) \subseteq C(\mathbb{H}) \otimes_{\min } C(\mathbb{H})$ and that $U$ is invariant under $\mathcal{H}^{\mathrm{CAP}} \otimes L^{2}(\mathbb{G})$. Taking $\zeta \in \mathcal{H}^{\mathrm{CP}}$ and letting $u$ be as in Definition 3.7, the elements $\left(\omega_{\zeta, \eta} \otimes \mathrm{id}\right)(U)=\left(\eta^{*} \otimes \mathrm{id}\right) \tilde{\alpha}(\zeta), \eta \in \mathcal{H}$, are just linear combinations of the matrix elements of $u$. Since the co-representation $u$ and its transpose $u^{\mathrm{t}}$ are invertible and the unital $C^{*}$-algebra $C(\mathbb{H})$ is generated by $\left\{\left(\omega_{\zeta, \eta} \otimes \mathrm{id}\right)(U): \zeta \in \mathcal{H}^{\mathrm{CP}}, \eta \in \mathcal{H}\right\}, \mathbb{H}$ is a compact quantum group by Maes and Van Daele [25, Proposition 3.8] (see also Woronowicz [39]). If $\mathbb{G}$ is a LCQG, then $C(\mathbb{H}) \subseteq M\left(C_{0}(\mathbb{G})\right.$ ) by Proposition 3.6 as $\mathcal{H}^{\mathrm{CAP}}$ is contained in the subspace $\mathcal{K}$ therein.

The restriction $V:=\left.U\right|_{\mathcal{H}_{\mathrm{CAP}} \otimes L^{2}(\mathbb{G})}$ is now a co-representation of $\mathbb{G}$ on $\mathcal{H}^{\mathrm{CAP}}$. By definition, $V$ (which is isometric) has dense range, so it is unitary. So we need only establish that $V \in$ $M\left(\mathbb{K}\left(\mathcal{H}^{\mathrm{CAP}}\right) \otimes_{\min } C(\mathbb{H})\right)$. Let $\zeta \in \mathcal{H}^{\mathrm{CP}}, \eta \in \mathcal{H}^{\mathrm{CAP}}$ and $x \in C(\mathbb{H})$. Since $\tilde{\alpha}\left(\mathcal{H}^{\mathrm{CP}}\right) \subseteq \mathcal{H}^{\mathrm{CP}} \odot$ $C(\mathbb{H})$, we get $V\left(\left(\zeta \otimes \eta^{*}\right) \otimes x\right) \in \mathbb{K}\left(\mathcal{H}^{\mathrm{CAP}}\right) \odot C(\mathbb{H})$. Let $\mathcal{H}_{1}$ be the finite-dimensional subspace associated with $\zeta$ in Definition 3.7 and $p_{\mathcal{H}_{1}}$ the projection of $\mathcal{H}^{\mathrm{CAP}}$ onto $\mathcal{H}_{1}$. Since $V$ is invariant under $\mathcal{H}_{1} \otimes L^{2}(\mathbb{G})$ and $u:=\left.V\right|_{\mathcal{H}_{1} \otimes L^{2}(\mathbb{G})} \in B\left(\mathcal{H}_{1}\right) \odot C(\mathbb{H})$ is unitary, we have $\left(\left(\eta \otimes \zeta^{*}\right) \otimes x\right) V=$ $\left(\left(\eta \otimes \zeta^{*}\right) \otimes x\right) u\left(p_{\mathcal{H}_{1}} \otimes \mathbb{1}\right) \in \mathbb{K}\left(\mathcal{H}^{\mathrm{CAP}}\right) \odot C(\mathbb{H})$. The foregoing implies that $V(k \otimes x)$ and $(k \otimes x) V$ belong to $\mathbb{K}\left(\mathcal{H}^{\mathrm{CAP}}\right) \otimes_{\min } C(\mathbb{H})$ for every $k \in \mathbb{K}\left(\mathcal{H}^{\mathrm{CAP}}\right)$ and $x \in C(\mathbb{H})$, hence $V \in M\left(\mathbb{K}\left(\mathcal{H}^{\mathrm{CAP}}\right) \otimes_{\text {min }}\right.$ $C(\mathbb{H}))$ as desired.

Corollary 3.12. Every left- (or right-) invariant state of $\mathbb{G}$ is faithful on $C(\mathbb{H})$.

Proof. Let $m$ be a left-invariant state of $\mathbb{G}$. Since $\left.m\right|_{C(\mathbb{I I})}$ is a left-invariant state of the compact quantum group $\mathbb{H}$, it must be equal to the Haar state of $\mathbb{H}[40,25]$, which is faithful as $\mathbb{H}$ is co-amenable [3, Theorem 2.2]. 
Under mild assumptions, we can provide another characterization of $\mathcal{H}^{\mathrm{CAP}}$, one which bears a stronger resemblance to the definition of almost periodicity used in [27, 41] for the classical case (approach (iii) above).

Definition 3.13. We say that a quantum semigroup $\mathbb{G}$ satisfies condition (I) if every isometric or co-isometric finite-dimensional co-representation of $\mathbb{G}$ is unitary.

Condition (I) is satisfied when $\mathbb{G}$ is a LCQG, in which case every isometric or co-isometric co-representation of $\mathbb{G}$ is unitary by [7, Corollaries 4.11, 4.12]. It is also satisfied when $L^{\infty}(\mathbb{G})$ is a finite von Neumann algebra.

Corollary 3.14. Let $\mathcal{H}^{\mathrm{CP} 2}$ be the set of all vectors $\zeta \in \mathcal{H}$ satisfying the following conditions:

(a) there exists a finite-dimensional sub-representation of $U$ on a space that contains $\zeta{ }^{2}$ and

(b) for every $\eta \in \mathcal{H}$ with $x:=\left(\omega_{\zeta, \eta} \otimes \mathrm{id}\right)(U) \neq 0$, we have $m\left(x^{*} x\right)>0$ for some left-invariant mean $m$ on $\mathbb{G}$.

We have $\mathcal{H}^{\mathrm{CP}} \subseteq \mathcal{H}^{\mathrm{CP} 2}$. If $\mathbb{G}$ satisfies condition (I), then $\mathcal{H}^{\mathrm{CP}}=\mathcal{H}^{\mathrm{CP} 2}$, so that $\overline{\mathrm{span}} \mathcal{H}^{\mathrm{CP} 2}=\mathcal{H}^{\mathrm{CAP}}$.

Condition (b) originates in a concrete interpretation of periodicity. In the setting of Niculescu, Ströh and Zsidó [27], one has the following: a vector $\zeta \in \mathcal{H}$ is almost periodic if and only if for every $\varepsilon>0$, the set $\left\{n \in \mathbb{Z}_{+}:\left\|U^{n} \zeta-\zeta\right\|<\varepsilon\right\}$ is relatively dense in $\mathbb{Z}_{+}$[27, Corollary 9.10]. This obviously implies that if $\zeta \in \mathcal{H}$ is almost periodic and $\eta \in \mathcal{H}$ is such that the function $x: \mathbb{Z}_{+} \rightarrow \mathbb{C}, n \mapsto\left\langle U^{n} \zeta, \eta\right\rangle$, is not identically zero, then $m\left(|x|^{2}\right)>0$ for every invariant mean $m$ on $\mathbb{Z}_{+}$. A similar assertion can be stated in the more general setting of Zsidó [41] as well. Thus, (b) can be viewed as a weak type of recurrence (which is automatic in the classical setting).

Proof of Corollary 3.14. If $\zeta \in \mathcal{H}^{\mathrm{CP}}$ and $\eta \in \mathcal{H}$ are such that $x:=\left(\omega_{\zeta, \eta} \otimes \mathrm{id}\right)(U) \neq 0$, then $m\left(x^{*} x\right)>0$ for every left- (or right-) invariant mean $m$ on $\mathbb{G}$ by Corollary 3.12 as $x \in C(\mathbb{H})$. In conclusion, $\mathcal{H}^{\mathrm{CP}} \subseteq \mathcal{H}^{\mathrm{CP} 2}$.

Let $\zeta \in \mathcal{H}^{\mathrm{CP} 2}$ with $\|\zeta\|=1$. Write $\tilde{\alpha}(\zeta)=\zeta_{1} \otimes a_{1}+\ldots+\zeta_{n} \otimes a_{n}$, where $a_{1}, \ldots, a_{n}$ are linearly independent and $\zeta_{1}, \ldots, \zeta_{n}$ are orthonormal. Let $\mathcal{H}_{1}:=\operatorname{span}\left\{\zeta_{1}, \ldots, \zeta_{n}\right\}$. By co-amenability, $\zeta \in \mathcal{H}_{1}$ (see Lemma 1.9), so we may assume that $\zeta=\zeta_{1}$.

For each $1 \leq i \leq n$, fix $\omega_{i} \in L^{1}(\mathbb{G})$ with $\omega_{i}\left(a_{j}\right)=\delta_{i j}$ for all $1 \leq j \leq n$. Now $\tilde{\alpha}\left(\zeta_{i}\right)=$ $\tilde{\alpha}\left(\left(\mathrm{id} \otimes \omega_{i}\right) \tilde{\alpha}(\zeta)\right)=\left(\mathrm{id} \otimes \mathrm{id} \otimes \omega_{i}\right)(\tilde{\alpha} \otimes \mathrm{id}) \tilde{\alpha}(\zeta)=\left(\mathrm{id} \otimes\left(\mathrm{id} \otimes \omega_{i}\right) \Delta\right) \tilde{\alpha}(\zeta)$. Hence $\mathcal{H}_{1}$ is invariant under $U$. Let $u \in B\left(\mathcal{H}_{1}\right) \odot L^{\infty}(\mathbb{G})$ be the co-representation of $\mathbb{G}$ on $\mathcal{H}_{1}$ given by $u:=\left.U\right|_{\mathcal{H}_{1} \otimes L^{2}(\mathbb{G})}$. Since $u$ is an isometry and $\mathbb{G}$ satisfies condition (I), $u$ is unitary. Write $u$ as a matrix $\left(u_{i j}\right)_{i, j=1}^{n} \in M_{n}\left(L^{\infty}(\mathbb{G})\right)$ by setting $u_{i j}:=\left(\zeta_{i}^{*} \otimes \mathrm{id}\right) \tilde{\alpha}\left(\zeta_{j}\right)$. In particular, $a_{i}=u_{i 1}$ for every $i$.

We need only show that $u^{\mathrm{t}}$ is invertible to establish that $\zeta \in \mathcal{H}^{\mathrm{CP}}$. Let $m$ be a left-invariant mean on $\mathbb{G}$ as in the corollary's statement. Since $u$ is a co-representation of $\mathbb{G}$,

$$
m\left(a_{i}^{*} a_{j}\right) \mathbb{1}=(\mathrm{id} \otimes m) \Delta\left(a_{i}^{*} a_{j}\right)=\sum_{k, l=1}^{n} u_{i k}^{*} u_{j l} m\left(a_{k}^{*} a_{l}\right) \quad(\forall 1 \leq i, j \leq n) .
$$

That is, letting $g:=\left(m\left(a_{k}^{*} a_{l}\right)\right)_{k, l}$, we get $u^{\mathrm{t} *}(g \otimes \mathbb{1}) u^{\mathrm{t}}=g \otimes \mathbb{1}$. The positive semi-definite matrix $g$ is invertible, for otherwise there is $0 \neq c=\left(c_{i}\right) \in \mathbb{C}^{n}$ such that $g c=0$. Thus, writing $x:=$

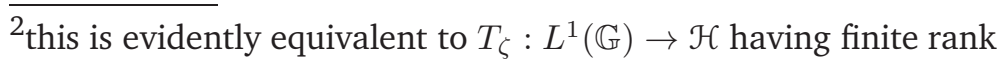


$\sum_{k=1}^{n} c_{k} a_{k}=\left(\left(\sum_{k=1}^{n} \overline{c_{k}} \zeta_{k}\right)^{*} \otimes \mathrm{id}\right) \tilde{\alpha}(\zeta)$, we have $x \neq 0$ (because $a_{1}, \ldots, a_{n}$ are linearly independent) but $m\left(x^{*} x\right)=0$, a contradiction. Consequently, $\left(g^{-1 / 2} \otimes \mathbb{1}\right) u^{t *}\left(g^{1 / 2} \otimes \mathbb{1}\right)$ is a co-isometric (finitedimensional) co-representation of $\mathbb{G}$. Hence, by condition (I), it is invertible. Therefore, so is $u^{\mathrm{t}}$. This completes the proof.

\section{ANALYSIS OF $\mathcal{H}^{\mathrm{CAP}}$ AND $N^{\mathrm{CAP}}$}

In this section we settle affirmatively Questions A-C (the last one under additional conditions). The two main tools are the compactification result of $\S 3$ and modular theory. Some of the results and techniques should be compared to Boca [6].

Fix a complete family $\left(u^{\gamma}\right)_{\gamma \in \operatorname{Irred}(\mathbb{H})}$ of irreducible unitary co-representations of $\mathbb{H}$ (see [40] for details). For each $\gamma \in \operatorname{Irred}(\mathbb{H}), u^{\gamma}=\left(u_{i j}^{\gamma}\right)_{i, j=1}^{n(\gamma)}$ belongs to $M_{n(\gamma)} \odot C(\mathbb{H})$. As $\mathbb{H}$ is a compactification of $\mathbb{G}$, every such $u^{\gamma}$ is also a co-representation of $\mathbb{G}$. Consider the construction in Theorem 2.2 with $C$ being $M_{n(\gamma)}$ and $\mu$ being the normalized trace $\operatorname{tr}_{\gamma}$ on $M_{n(\gamma)}$. Denoting the GNS construction of $\left(M_{n(\gamma)}, \operatorname{tr}_{\gamma}\right)$ by $\left(\mathcal{H}_{\gamma}, \Gamma_{\gamma}\right)$, we get the associated projection $P_{u^{\gamma}} \in B\left(\mathcal{H}_{\gamma} \otimes \mathcal{H}\right)$ and normal projection $E_{u^{\gamma}}: M_{n(\gamma)} \odot N \rightarrow N_{u^{\gamma}}=\left\{b \in M_{n(\gamma)} \odot N:(\right.$ id $\left.\otimes \alpha)(b)=u_{13}^{\gamma *} b_{12}\right\}$. Write $\left(e_{i j}^{\gamma}\right)$ for a system of matrix units for $M_{n(\gamma)}$.

Lemma 4.1. We have $\operatorname{Im} P_{u^{\gamma}} \subseteq \mathcal{H}_{\mu} \otimes \mathcal{H}^{\mathrm{CAP}}$ and $N_{u^{\gamma}}=\operatorname{Im} E_{u^{\gamma}} \subseteq M_{n(\gamma)} \odot N^{\mathrm{CAP}}$ for all $\gamma \in \operatorname{Irred}(\mathbb{H})$. Proof. Write $u, n$ for $u^{\gamma}, n(\gamma)$, respectively. Let $b \in \operatorname{Im} E_{u}$. For all $1 \leq i, j \leq n$, we have $\alpha\left(b_{i j}\right)=$ $\sum_{k=1}^{n} b_{k j} \otimes\left(u^{\gamma *}\right)_{i k}$, hence $\tilde{\alpha}\left(\Gamma\left(b_{i j}\right)\right)=\sum_{k=1}^{n} \Gamma\left(b_{k j}\right) \otimes\left(u^{\gamma *}\right)_{i k}$. Fix $j$. The operators $b_{i j}, 1 \leq i \leq n$, are linearly independent unless all zero, as $u$ is irreducible. Since $u^{\mathrm{t}}$ is invertible [40, 25], the sub-representation of $U$ on span $\left\{\Gamma\left(b_{i j}\right)\right\}_{i=1}^{n}$ satisfies the condition of Definition 3.7, so that $\Gamma\left(b_{i j}\right) \in \mathcal{H}^{\mathrm{CP}}$, thus $b_{i j} \in N^{\mathrm{CAP}}$, for every $i$. This proves the second assertion. The first one follows by Theorem 2.2, (b).

Lemma 4.2. For every $a \in N^{\mathrm{CAP}}$ and $\rho \in N_{*}$ we have $(\rho \otimes \mathrm{id}) \alpha(a) \in C(\mathbb{H})$.

Proof. If $\rho$ is of the form $\rho(x)=\langle\Gamma(x), \eta\rangle$ for some $\eta \in \mathcal{H}$, then $(\rho \otimes$ id $) \alpha(a)=\left(\eta^{*} \otimes\right.$ id $) \tilde{\alpha}(\Gamma(a)) \in$ $C(\mathbb{H})$ by construction. The Hahn-Banach theorem implies that the subspace of functionals of this form is norm dense in $N_{*}$, and the assertion follows.

Let now $A_{u^{\gamma}}\left(\subseteq N^{\mathrm{CAP}}\right)$ denote the "right leg of $N_{u^{\gamma}}=\operatorname{Im} E_{u u^{\gamma}}$ ", namely the span of all matrix elements of matrices in $\operatorname{Im} E_{u^{\gamma}}$. Similarly, let $B_{u^{\gamma}}\left(\subseteq \mathcal{H}^{\mathrm{CAP}}\right)$ denote the "right leg of $\operatorname{Im} P_{u \gamma}$ ".

Proposition 4.3. The following assertions hold.

(a) The set $\bigcup_{\gamma \in \operatorname{Irred}(\mathbb{H})} B_{u^{\gamma}}$ is total in $\mathcal{H}^{\mathrm{CAP}}$.

(b) The set $\Gamma\left(\bigcup_{\gamma \in \operatorname{Irred}(\mathbb{H I})} A_{u^{\gamma}}\right)$ is total in $\mathcal{H}^{\mathrm{CAP}}$.

(c) $\overline{\Gamma\left(N^{\mathrm{CAP}}\right)}=\mathcal{H}^{\mathrm{CAP}}$ (Question B).

(d) The set $\bigcup_{\gamma \in \operatorname{Irred}(\mathbb{H})} A_{u^{\gamma}}$ is weakly total in $N^{\mathrm{CAP}}$.

Proof. Let $\eta \in \mathcal{H}$ be orthogonal to $\bigcup_{\gamma \in \operatorname{Irred}(\mathbb{H})} B_{u^{\gamma}}$. Let $m$ be an invariant mean on $\mathbb{G}$, and fix $\left(m_{\kappa}\right)$ as in Remark 1.5. For all $\zeta \in \mathcal{H}^{\mathrm{CAP}}, \gamma \in \operatorname{Irred}(\mathbb{H})$ and $1 \leq i, j \leq n(\gamma)$ we have, by Theorem 2.2,

$$
0=\eta^{*}\left(\left(\Gamma_{\gamma}\left(e_{i j}^{\gamma}\right)^{*} \otimes \mathrm{id}\right) P_{u^{\gamma}}\left(\Gamma_{\gamma}(\mathbb{1}) \otimes \zeta\right)\right)=\lim _{\lambda} m_{\lambda}\left(u_{i j}^{\gamma} \cdot\left(\eta^{*} \otimes \mathrm{id}\right) \tilde{\alpha}(\zeta)\right)=m\left(u_{i j}^{\gamma} \cdot\left(\eta^{*} \otimes \mathrm{id}\right) \tilde{\alpha}(\zeta)\right) .
$$


Hence $x:=\left(\eta^{*} \otimes \mathrm{id}\right) \tilde{\alpha}(\zeta)$ belongs to $C(\mathbb{H})$ and satisfies $m\left(u_{i j}^{\gamma} x\right)=0$. Since the span of all $u_{i j}^{\gamma}$ for $\gamma \in \operatorname{Irred}(\mathbb{H}), 1 \leq i, j \leq n(\gamma)$, is dense in $C(\mathbb{H})$, we have $m\left(x^{*} x\right)=0$ which, by Corollary 3.12, implies that $x=0$. By co-amenability of $\mathbb{G}$, we get

$$
0=\lim _{\lambda} \epsilon_{\lambda}\left[\left(\eta^{*} \otimes \mathrm{id}\right) \tilde{\alpha}(\zeta)\right]=\lim _{\lambda}\left\langle\left(\mathrm{id} \otimes \epsilon_{\lambda}\right) \tilde{\alpha}(\zeta), \eta\right\rangle=\langle\zeta, \eta\rangle
$$

for all $\zeta \in \mathcal{H}^{\mathrm{CAP}}$ (see Lemma 1.9). This proves (a) by Lemma 4.1.

From Theorem 2.2, (b) we know that $\overline{\Gamma\left(A_{u^{\gamma}}\right)}=B_{u^{\gamma}}$ for all $\gamma \in \operatorname{Irred}(\mathbb{H})$, so (b) follows. This in turn implies (c) because $\bigcup_{\gamma \in \operatorname{Irred}(\mathbb{H})} A_{u^{\gamma}} \subseteq N^{\mathrm{CAP}}$ (Lemma 4.1).

The proof of (d) is very similar to that of (a). Suppose that $\rho \in N_{*}$ vanishes on $A_{u^{\gamma}}$ for every $\gamma \in \operatorname{Irred}(\mathbb{H})$. This means that for all $a \in N^{\mathrm{CAP}}, \gamma \in \operatorname{Irred}(\mathbb{H})$ and $1 \leq i, j \leq n(\gamma)$, we have $m\left(u_{i j}^{\gamma} \cdot(\rho \otimes \mathrm{id}) \alpha(a)\right)=0$. From Lemma 4.2 we obtain $x:=(\rho \otimes \mathrm{id}) \alpha(a) \in C(\mathbb{H})$. As above, we infer that $x=0$, and thus $\rho(a)=0$ by co-amenability.

Henceforth we denote the (faithful) Haar state of $C(\mathbb{H})$ by $h$. The notations $\sigma^{h}$ and $\sigma^{\omega}$ stand for the modular automorphism groups of $h$ and $\omega$, respectively. We write $\bar{u}$ for the contragradient to a unitary irreducible co-representation $u$ of $\mathbb{H}$, that is, the unique element of $\left(u^{\gamma}\right)_{\gamma \in \operatorname{Irred}(\mathbb{H})}$ that is equivalent to $u^{* t}$. (Note that $u^{* t}$ is denoted by $\bar{u}$ in [25].)

Proposition 4.4. For every $\gamma, \delta \in \operatorname{Irred}(\mathbb{H})$ we have

(a) $A_{u^{\gamma}}^{*}=A_{\overline{u^{\gamma}}}$;

(b) $A_{u^{\gamma}} A_{u^{\delta}} \subseteq \operatorname{span}_{\beta \in \operatorname{Irred}(\mathbb{H})} A_{u^{\beta}}$.

Proof. (a) Write $u, n$ for $u^{\gamma}, n(\gamma)$, respectively, and let $a=\left(a_{i j}\right)_{i, j=1}^{n} \in M_{n} \odot N$. Since $E_{u}$ is a projection, we may assume, for the purpose of showing that the matrix elements of $E_{u}(a)^{*}$ belong to $A_{\bar{u}}$, that $a \in \operatorname{Im} E_{u}$. Therefore $\alpha\left(a_{i j}\right) \in N \odot C(\mathbb{H})$ for every $i, j$ (Lemmas 4.1 and 4.2). Let $m$ be an invariant mean on $\mathbb{G}$. Recalling that $h=\left.m\right|_{C(\mathbb{H})}$ (see proof of Corollary 3.12), we have, by Theorem 2.2 and Remark 1.5,

$$
E_{u}(a)^{*}=\left\{(\mathrm{id} \otimes \mathrm{id} \otimes h)\left[u_{13} \cdot(\mathrm{id} \otimes \alpha)(a)\right]\right\}^{*}=(\mathrm{id} \otimes \mathrm{id} \otimes h)\left[(\mathrm{id} \otimes \alpha)\left(a^{*}\right) \cdot u_{13}^{*}\right] .
$$

Let $E, F \in G L_{n}, F$ positive definite, satisfy $\bar{u}=(E \otimes \mathbb{1}) u^{* \mathrm{t}}\left(E^{-1} \otimes \mathbb{1}\right)$ and $\left(\mathrm{id} \otimes \sigma_{i}^{h}\right) \bar{u}=(F \otimes$ $\mathbb{1}) \bar{u}(F \otimes \mathbb{1})($ see $[40])$. Then $\left(\mathrm{id} \otimes \sigma_{i}^{h}\right)\left(u^{*}\right)=\left[\left(E^{-1} \otimes \mathbb{1}\right)\left(\mathrm{id} \otimes \sigma_{i}^{h}\right)(\bar{u})(E \otimes \mathbb{1})\right]^{\mathrm{t}}$, and therefore

$$
\begin{aligned}
E_{u}(a)^{*} & =\sum_{i, j, k=1}^{n} e_{i j} \otimes(\mathrm{id} \otimes h)\left(\alpha\left(a_{k i}^{*}\right)\left(\mathbb{1} \otimes u_{j k}^{*}\right)\right) \\
& =\sum_{i, j, k=1}^{n} e_{i j} \otimes(\mathrm{id} \otimes h)\left\{\left[\mathbb{1} \otimes\left(\left(E^{-1} F \otimes \mathbb{1}\right) \bar{u}(F E \otimes \mathbb{1})\right)_{j k}\right] \alpha\left(a_{k i}^{*}\right)\right\}
\end{aligned}
$$

by the Tomita-Takesaki theory. Denoting $G_{1}:=E^{-1} F$ and $G_{2}:=F E$, we get

$$
\begin{aligned}
E_{u}(a)^{* \mathrm{t}} & =(\mathrm{id} \otimes \mathrm{id} \otimes h)\left(\left(G_{1} \otimes \mathbb{1} \otimes \mathbb{1}\right) \bar{u}_{13}\left(G_{2} \otimes \mathbb{1} \otimes \mathbb{1}\right)(\mathrm{id} \otimes \alpha)\left(a^{* \mathrm{t}}\right)\right) \\
& =\left(G_{1} \otimes \mathbb{1}\right)(\mathrm{id} \otimes \mathrm{id} \otimes h)\left[\bar{u}_{13}(\mathrm{id} \otimes \alpha)\left(\left(G_{2} \otimes \mathbb{1}\right) a^{* \mathrm{t}}\right)\right] \\
& =\left(G_{1} \otimes \mathbb{1}\right) E_{\bar{u}}\left(\left(G_{2} \otimes \mathbb{1}\right) a^{* \mathrm{t}}\right) .
\end{aligned}
$$


This implies that $A_{u}^{*} \subseteq A_{\bar{u}}$. The converse inclusion is obtained by interchanging the roles of $u$ and $\bar{u}$.

(b) Given $a \in \operatorname{Im} E_{u^{\gamma}}$ and $b \in \operatorname{Im} E_{u^{\delta}}$, that is, (id $\left.\otimes \alpha\right)(a)=u_{13}^{\gamma *} a_{12}$ and (id $\left.\otimes \alpha\right)(b)=u_{13}^{\delta *} b_{12}$, we get

$$
\left(\operatorname{id}_{M_{n(\gamma)}} \otimes \operatorname{id}_{M_{n(\delta)}} \otimes \alpha\right)\left(a_{13} b_{23}\right)=u_{14}^{\gamma *} a_{13} u_{24}^{\delta *} b_{23}=u_{14}^{\gamma *} u_{24}^{\delta *} a_{13} b_{23}
$$

Thus, for $c:=a_{13} b_{23} \in M_{n(\gamma)} \odot M_{n(\delta)} \odot N$ and the unitary co-representation $u:=u_{23}^{\delta} u_{13}^{\gamma}$ of $\mathbb{H}$, we have $c \in \operatorname{Im} E_{u}$. Since $u$ decomposes (with respect to some basis) as $u=u^{\beta_{1}} \oplus \ldots \oplus u^{\beta_{n}}$ for suitable $\beta_{1}, \ldots, \beta_{n} \in \operatorname{Irred}(\mathbb{H})$ [40], the desired conclusion follows.

Theorem 4.5. The space $N^{\mathrm{CAP}}$ is a von Neumann subalgebra of $N$ (Question A).

Proof. The subspace span $\bigcup_{\gamma \in \operatorname{Irred}(\mathbb{H})} A_{u^{\gamma}}$ is a $*$-subalgebra of $N^{\mathrm{CAP}}$ by Proposition 4.4. Thus the assertion follows from Proposition 4.3, (d).

We now address Question C, providing a few circumstances under which it has an affirmative answer.

Theorem 4.6. The von Neumann algebra $N^{\mathrm{CAP}}$ is globally invariant under $\sigma^{\omega}$ if $\mathbb{G}$ is a LCQG.

Proof. Denote by $\tau^{\mathbb{G}}, \tau^{\mathbb{H}}$ the scaling groups of $\mathbb{G}, \mathbb{H}$, respectively. Since $\alpha$ is an $\omega$-invariant action of $\mathbb{G}$ on $N$, we get, by Theorem A.1,

$$
\left(\sigma_{t}^{\omega} \otimes \tau_{-t}^{\mathbb{G}}\right) \circ \alpha=\alpha \circ \sigma_{t}^{\omega} \quad(\forall t \in \mathbb{R}) .
$$

Fix $\gamma \in \operatorname{Irred}(\mathbb{H})$ and denote $u:=u^{\gamma}$. There exists a positive definite $F \in G L_{n(\gamma)}$ such that $\left(\mathrm{id} \otimes \tau_{t}^{\mathbb{H}}\right) u=\left(F^{i t} \otimes \mathbb{1}\right) u\left(F^{-i t} \otimes \mathbb{1}\right)$ for each $t \in \mathbb{R}$ [40]. Thus [23, Proposition 5.45] implies that $\left(\operatorname{id} \otimes \tau_{t}^{\mathbb{G}}\right) u=\left(F^{i t} \otimes \mathbb{1}\right) u\left(F^{-i t} \otimes \mathbb{1}\right)$. If $a \in \operatorname{Im} E_{u}$, namely $(\operatorname{id} \otimes \alpha)(a)=u_{13}^{*} a_{12}$, then for every $t \in \mathbb{R}$, we obtain

$$
\begin{aligned}
\left(\mathrm{id} \otimes \alpha \circ \sigma_{t}^{\omega}\right)(a) & =\left(\mathrm{id} \otimes\left(\sigma_{t}^{\omega} \otimes \tau_{-t}^{\mathbb{G}}\right) \circ \alpha\right)(a)=\left(\mathrm{id} \otimes \sigma_{t}^{\omega} \otimes \tau_{-t}^{\mathbb{G}}\right)\left(u_{13}^{*} a_{12}\right) \\
& =\left(F^{-i t} \otimes \mathbb{1} \otimes \mathbb{1}\right) u_{13}^{*}\left(F^{i t} \otimes \mathbb{1} \otimes \mathbb{1}\right)\left(\left(\mathrm{id} \otimes \sigma_{t}^{\omega}\right)(a)\right)_{12} .
\end{aligned}
$$

Therefore

$$
(\mathrm{id} \otimes \alpha)\left[\left(\mathrm{id} \otimes \sigma_{t}^{\omega}\right)\left(\left(F^{i t} \otimes \mathbb{1}\right) a\right)\right]=u_{13}^{*}\left[\left(\mathrm{id} \otimes \sigma_{t}^{\omega}\right)\left(\left(F^{i t} \otimes \mathbb{1}\right) a\right)\right]_{12},
$$

proving that $\left(\mathrm{id} \otimes \sigma_{t}^{\omega}\right)\left(\left(F^{i t} \otimes \mathbb{1}\right) a\right) \in \operatorname{Im} E_{u}$. Thus $A_{u}$ is globally invariant under $\sigma^{\omega}$. The result follows from Proposition 4.3, (d).

Theorem 4.7. The von Neumann algebra $N^{\mathrm{CAP}}$ is globally invariant under $\sigma^{\omega}$ if the following is true: for every $\gamma \in \operatorname{Irred}(\mathbb{H})$ there exists an invariant mean $m$ on $\mathbb{G}$ such that for each $1 \leq i, j \leq n(\gamma)$ there is $v \in C(\mathbb{H})$ with $m\left(x u_{i j}^{\gamma}\right)=m(v x)$ for all $x \in L^{\infty}(\mathbb{G})$.

Of course, this condition holds trivially if $\mathbb{G}$ is classical, namely $L^{\infty}(\mathbb{G})$ is commutative.

Proof. First, note that $v=\sigma_{i}^{h}\left(u_{i j}^{\gamma}\right)$ in the theorem's condition by Corollary 3.12. We use the notation and results of Proposition 4.4. Fix $\gamma \in \operatorname{Irred}(\mathbb{H})$, and write $u, n$ for $u^{\gamma}, n(\gamma)$, respectively. Let $m$ be the invariant mean suitable for $\bar{u}$ as above. In the proof of Proposition 4.4, (a), we made the assumption that $a \in \operatorname{Im} E_{u}$. This cannot be done in the present proof. Taking an 
arbitrary $a=\left(a_{i j}\right)_{i, j=1}^{n} \in M_{n} \odot N$, we repeat the calculations of Proposition 4.4 with $m$ replacing $h$. The maneuver of (4.1) is now justified by the assumption on $m$, and we infer from (4.2) that $E_{u}(a)^{* t}=\left(G_{1} \otimes \mathbb{1}\right) E_{\bar{u}}\left(\left(G_{2} \otimes \mathbb{1}\right) a^{* \mathrm{t}}\right)$.

Let $J, \nabla$ denote the modular conjugation and modular operator, respectively, associated with $(N, \omega)$. That is, the closure $S$ of the conjugate-linear map $\Gamma(x) \mapsto \Gamma\left(x^{*}\right), x \in N$, over $\mathcal{H}$, has polar decomposition $S=J \nabla^{1 / 2}$. Write $A$ for the componentwise complex conjugation map $\Gamma_{\gamma}\left(\left(a_{i j}\right)\right) \mapsto \Gamma_{\gamma}\left(\left(\overline{a_{i j}}\right)\right)$ over $\mathcal{H}_{\gamma}$. Then by the previous paragraph and Theorem 2.2,

$$
\begin{aligned}
(A \otimes S) P_{u} \Xi(a) & =(A \otimes S) \Xi\left(E_{u}(a)\right)=\Xi\left(E_{u}(a)^{* \mathrm{t}}\right) \\
& =\Xi\left[\left(G_{1} \otimes \mathbb{1}\right) E_{\bar{u}}\left(\left(G_{2} \otimes \mathbb{1}\right) a^{* \mathrm{t}}\right)\right]=\left(G_{1} \otimes \mathbb{1}\right) \Xi\left[E_{\bar{u}}\left(\left(G_{2} \otimes \mathbb{1}\right) a^{* \mathrm{t}}\right)\right] \\
& =\left(G_{1} \otimes \mathbb{1}\right) P_{\bar{u}}\left(\left(G_{2} \otimes \mathbb{1}\right) \Xi\left(a^{* \mathrm{t}}\right)\right)=\left(G_{1} \otimes \mathbb{1}\right) P_{\bar{u}}\left(\left(G_{2} \otimes \mathbb{1}\right)(A \otimes S) \Xi(a)\right)
\end{aligned}
$$

for all $a \in M_{n} \odot N$. Therefore

$$
\left(G_{1} \otimes \mathbb{1}\right) P_{\bar{u}}\left(G_{2} \otimes \mathbb{1}\right)(A \otimes S) \subseteq(A \otimes S) P_{u} .
$$

As $S=J \nabla^{1 / 2}=\nabla^{-1 / 2} J$, we have

$$
\left(G_{1} \otimes \mathbb{1}\right) P_{\bar{u}}\left(G_{2} \otimes \mathbb{1}\right)\left(A \otimes \nabla^{-1 / 2} J\right) \subseteq\left(A \otimes \nabla^{-1 / 2} J\right) P_{u},
$$

and thus

$$
P_{\bar{u}}\left(\mathbb{1} \otimes \nabla^{-1 / 2}\right) \subseteq\left(\mathbb{1} \otimes \nabla^{-1 / 2}\right)\left(G_{1}^{-1} \otimes \mathbb{1}\right)(A \otimes J) P_{u}(A \otimes J)\left(G_{2}^{-1} \otimes \mathbb{1}\right) .
$$

Recall that $\sigma_{t}^{\omega}=\left.\operatorname{Ad}\left(\nabla^{i t}\right)\right|_{N} \in \operatorname{Aut}(N)$ for all $t \in \mathbb{R}$. Abusing notation slightly and letting $\sigma_{t}^{\omega}:=\operatorname{Ad}\left(\nabla^{i t}\right) \in \operatorname{Aut}(B(\mathcal{H}))$ for $t \in \mathbb{R}$, we deduce that

$$
P_{\bar{u}} \in D\left(\mathrm{id} \otimes \sigma_{-i / 2}^{\omega}\right) \quad \text { and } \quad\left(\mathrm{id} \otimes \sigma_{-i / 2}^{\omega}\right)\left(P_{\bar{u}}\right)=\left(G_{1}^{-1} \otimes \mathbb{1}\right)(A \otimes J) P_{u}(A \otimes J)\left(G_{2}^{-1} \otimes \mathbb{1}\right)
$$

(see [8, Theorem 6.2]). As $P_{u}, P_{\bar{u}}$ are selfadjoint, using (4.3) twice we obtain $P_{\bar{u}} \in D\left(\mathrm{id} \otimes \sigma_{i / 2}^{\omega}\right)$ and

$$
\begin{aligned}
(\mathrm{id} \otimes & \left.\sigma_{i / 2}^{\omega}\right)\left(P_{\bar{u}}\right)=\left[\left(\mathrm{id} \otimes \sigma_{-i / 2}^{\omega}\right)\left(P_{\bar{u}}\right)\right]^{*}=\left(G_{2}^{-1 *} \otimes \mathbb{1}\right)(A \otimes J) P_{u}(A \otimes J)\left(G_{1}^{-1 *} \otimes \mathbb{1}\right) \\
& =\left(G_{2}^{-1 *} \otimes \mathbb{1}\right)(A \otimes J)(A \otimes J)\left(G_{1} \otimes \mathbb{1}\right)\left(\mathrm{id} \otimes \sigma_{-i / 2}^{\omega}\right)\left(P_{\bar{u}}\right)\left(G_{2} \otimes \mathbb{1}\right)(A \otimes J)(A \otimes J)\left(G_{1}^{-1 *} \otimes \mathbb{1}\right) \\
& =\left(G_{2}^{-1 *} G_{1} \otimes \mathbb{1}\right)\left(\mathrm{id} \otimes \sigma_{-i / 2}^{\omega}\right)\left(P_{\bar{u}}\right)\left(G_{2} G_{1}^{-1 *} \otimes \mathbb{1}\right) .
\end{aligned}
$$

This entails that $P_{\bar{u}} \in D\left(\mathrm{id} \otimes \sigma_{i}^{\omega}\right)$ and

$$
\left(\mathrm{id} \otimes \sigma_{i}^{\omega}\right)\left(P_{\bar{u}}\right)=\left(G_{2}^{-1 *} G_{1} \otimes \mathbb{1}\right) P_{\bar{u}}\left(G_{2} G_{1}^{-1 *} \otimes \mathbb{1}\right) .
$$

Since $u^{* \mathrm{t}}$ is a (normally not unitary) finite-dimensional co-representation of $\mathbb{H}$, from the proof of [25, Proposition 6.4] follows the existence of a positive definite $F \in G L_{n}$ such that $w:=$ $\left(F^{1 / 2} \otimes \mathbb{1}\right) u^{* \mathrm{t}}\left(F^{-1 / 2} \otimes \mathbb{1}\right)$ is a unitary co-representation of $\mathbb{H}$ (which is equivalent to $\bar{u}$ ) and $\left(\right.$ id $\left.\otimes \sigma_{i}^{h}\right) w=(F \otimes \mathbb{1}) w(F \otimes \mathbb{1})$. Therefore, replacing $\bar{u}$ by an equivalent unitary co-representation of $\mathbb{H}$ if needed, we may assume that $E=F^{1 / 2}$ in the notation of the proof of Proposition 4.4, (a). Thus $G_{1}=F^{1 / 2}$ and $G_{2}=F^{3 / 2}$, so that $G_{2}^{-1 *} G_{1}=F^{-1}=\left(G_{2} G_{1}^{-1 *}\right)^{-1}$. As a result,

$$
\left(\mathrm{id} \otimes \sigma_{i}^{\omega}\right)\left(P_{\bar{u}}\right)=\left(F^{-1} \otimes \mathbb{1}\right) P_{\bar{u}}(F \otimes \mathbb{1}) .
$$


Consequently, $\left(\mathrm{id} \otimes \sigma_{t}^{\omega}\right)\left(P_{\bar{u}}\right)=\left(F^{i t} \otimes \mathbb{1}\right) P_{\bar{u}}\left(F^{-i t} \otimes \mathbb{1}\right)$ for every $t \in \mathbb{R}$. Thus, for every $a \in M_{n} \odot N$,

$$
\begin{aligned}
& \Xi E_{\bar{u}}\left(\left(\mathrm{id} \otimes \sigma_{t}^{\omega}\right)(a)\right)=P_{\bar{u}}\left(\mathbb{1} \otimes \nabla^{i t}\right) \Xi(a) \\
& \quad=\left(\mathbb{1} \otimes \nabla^{i t}\right)\left(F^{-i t} \otimes \mathbb{1}\right) P_{\bar{u}}\left(F^{i t} \otimes \mathbb{1}\right) \Xi(a)=\Xi\left(\mathrm{id} \otimes \sigma_{t}^{\omega}\right)\left(\left(F^{-i t} \otimes \mathbb{1}\right) E_{\bar{u}}\left(\left(F^{i t} \otimes \mathbb{1}\right) a\right)\right),
\end{aligned}
$$

and by injectivity of $\Xi,\left(F^{i t} \otimes \mathbb{1}\right) E_{\bar{u}}\left(\left(\operatorname{id} \otimes \sigma_{t}^{\omega}\right)(a)\right)=\left(\operatorname{id} \otimes \sigma_{t}^{\omega}\right) E_{\bar{u}}\left(\left(F^{i t} \otimes \mathbb{1}\right) a\right)$. Hence $\left(\operatorname{id} \otimes \sigma_{t}^{\omega}\right) E_{\bar{u}}(a)=$ $\left(F^{i t} \otimes \mathbb{1}\right) E_{\bar{u}}\left(\left(\right.\right.$ id $\left.\left.\otimes \sigma_{t}^{\omega}\right)\left(\left(F^{-i t} \otimes \mathbb{1}\right) a\right)\right)$ for all $a \in M_{n} \odot N$, and so $\sigma_{t}^{\omega}\left(A_{\bar{u}}\right) \subseteq A_{\bar{u}}$. The result follows from Proposition 4.3, (d).

Remark 4.8. The mean mentioned in Theorem 4.7 has the flavor of a hypertrace in the sense of Connes [9]. Obviously, an invariant mean $m$ on $\mathbb{G}$ admitting an $m$-preserving conditional expectation from $L^{\infty}(\mathbb{G})$ onto $C(\mathbb{H})$ satisfies the indicated condition for every $\gamma \in \operatorname{Irred}(\mathbb{H})$. But a conditional expectation from $L^{\infty}(\mathbb{G})$ onto $C(\mathbb{H})$ normally does not exist: for instance, taking $\mathbb{G}:=\mathbb{Z}_{+}$with an action yielding $\mathbb{H} \cong \mathbb{T}$, we would be looking for a conditional expectation from $\ell_{\infty}$ onto $c_{0}$, which does not exist as $c_{0}$ is not complemented in $\ell_{\infty}$. The existence of such a conditional expectation is, nevertheless, not necessary for the assumptions of Theorem 4.7 to hold. Notice further that we cannot replace $C(\mathbb{H})$ by $L^{\infty}(\mathbb{H})$, because even in the classical theory, the latter cannot normally be quantum embedded in $L^{\infty}(\mathbb{G})$.

Proposition 4.9. The condition of Theorem 4.7 holds if $\mathbb{H}$ is co-commutative, i.e., if it is the dual of a discrete group.

Proof. Let $m$ be any invariant mean on $\mathbb{G}$. Let $H$ be the discrete group so that $\mathbb{H}=\hat{H}$ (Example 1.6, (b)). Co-amenability of $\mathbb{H}$ is equivalent to amenability of $H$. Denote by $\left(\lambda_{h}\right)_{h \in H}$ the translation maps on $H$, and recall that $\Delta_{\hat{H}}\left(\lambda_{h}\right)=\lambda_{h} \otimes \lambda_{h}$ for every $h \in H$. Let $K$ stand for the $w^{*}$-compact convex subset of $L^{\infty}(\mathbb{G})^{*}$ consisting of all invariant means on $\mathbb{G}$. Consider the action of $H$ on $K$ given by $(h \cdot m)(x):=m\left(\lambda_{h} x \lambda_{h^{-1}}\right)\left(h \in H, m \in K, x \in L^{\infty}(\mathbb{G})\right)$. This action is indeed well defined, as for every $x \in L^{\infty}(\mathbb{G})$ and $\theta \in L^{1}(\mathbb{G})$ we have, by left invariance of $m$,

$$
\begin{aligned}
(h \cdot m)((\theta \otimes \mathrm{id}) \Delta(x)) & =m\left[\lambda_{h}(\theta \otimes \mathrm{id}) \Delta(x) \lambda_{h^{-1}}\right]=m\left\{(\theta \otimes \mathrm{id})\left[\left(\mathbb{1} \otimes \lambda_{h}\right) \Delta(x)\left(\mathbb{1} \otimes \lambda_{h^{-1}}\right)\right]\right\} \\
& =m\left\{(\theta \otimes \mathrm{id})\left[\left(\lambda_{h^{-1}} \otimes \mathbb{1}\right) \Delta\left(\lambda_{h} x \lambda_{h^{-1}}\right)\left(\lambda_{h} \otimes \mathbb{1}\right)\right]\right\} \\
& =m\left\{\left[\theta\left(\lambda_{h^{-1}} \cdot \lambda_{h}\right) \otimes \mathrm{id}\right] \Delta\left(\lambda_{h} x \lambda_{h^{-1}}\right)\right\} \\
& =\theta\left(\lambda_{h^{-1}} \cdot \lambda_{h}\right)(\mathbb{1}) m\left(\lambda_{h} x \lambda_{h^{-1}}\right)=\theta(\mathbb{1})(h \cdot m)(x) .
\end{aligned}
$$

Therefore, the mean $h \cdot m$ is left invariant, and similarly it is also right invariant. The action of $H$ on $K$ is plainly affine and separately continuous ( $H$ is discrete!). Thus, by Day's fixed point theorem [28, Theorem 1.3.1], there exists $m \in K$ with $h \cdot m=m$ for all $h \in H$, namely $m\left(\lambda_{h} x \lambda_{h^{-1}}\right)=m(x)$ for all $x \in L^{\infty}(\mathbb{G})$ and $h \in H$. As $\left(\lambda_{h}\right)_{h \in H}$ forms a complete family of irreducible unitary co-representations of $\mathbb{H}$, we are done.

Our ultimate purpose of giving a von Neumann algebraic version of the Jacobs-de LeeuwGlicksberg splitting theorem is achieved in terms of conditional expectations as follows. The precise nature of the image of id $-E^{\mathrm{CAP}}$, namely the weakly mixing operators, will be the subject of another paper. 
Corollary 4.10. Assume that either $\mathbb{G}$ is a LCQG or the condition of Theorem 4.7 holds. Then there exists a unique w-preserving conditional expectation $E^{\mathrm{CAP}}$ from $N$ onto $N^{\mathrm{CAP}}$. Moreover, $E^{\mathrm{CAP}}$ is faithful and normal, and denoting by $P^{\mathrm{CAP}}$ the projection of $\mathcal{H}_{\text {onto }} \mathcal{H}^{\mathrm{CAP}}$, we have $\Gamma \circ E^{\mathrm{CAP}}=P^{\mathrm{CAP}} \circ \Gamma$.

Proof. By virtue of Theorem 4.6 or Theorem 4.7 as well as Proposition 4.3, (c), the assertion follows from Takesaki's theorem and its proof ([33], see also [31, 34]).

Remark 4.11. If $N$ is finite and $\omega$ is a (finite) trace, the result of Corollary 4.10 holds trivially (by Takesaki's theorem) without any assumption on $\mathbb{G}$.

Problem 4.12. Determine when the condition of Theorem 4.7 holds. Specifically, does it hold when $\mathbb{G}$ is a LCQG?

Problem 4.13. Determine whether the generalized Day's fixed point theorem for amenable LCQGs (see [18, Théorème 2.4, (xii)] for Kac algebras; the proof for general LCQGs is identical) can be applied to produce a generalization of Proposition 4.9 for every possible $\mathbb{H}$, or at least when $\mathbb{H}$ is a Kac algebra (equivalently, $h$ is tracial).

\section{APPENDiX A. STATE-PRESERVING ACTIONS OF LCQGS}

The result that we prove in this appendix has appeared implicitly in several publications, as have some similar results. For instance, [36, Proposition 2.4] involves a $\delta^{-1}$-invariant weight and a corresponding co-representation; it is asserted that the proof is similar to that of [17, Théorème 2.9], although the second condition in [17, Définition 2.7] appears to be missing from [36, Definition 2.3]. As a second example, in the proof of [37, Theorem 2.11], the authors used (the $\mathbb{1}$-invariant, rather than $\delta^{-1}$-invariant, version of) [36, Proposition 4.3] to prove that their operator $\hat{Z}_{1}$ is the canonical implementing unitary of the action $\hat{\mu}$, without referring to the second condition in [17, Définition 2.7].

To conclude, Theorem A.1 is clearly known to the experts, but since we could not find an explicit reference, we include here the full proof for completeness. The ideas are by no means new: they are taken from Kustermans and Vaes [23, §5]. More general statements, related to the foregoing examples, can be proved in a similar fashion.

Theorem A.1. Let $\mathbb{G}$ be a LCQG, $N$ a von Neumann algebra, $\omega$ a faithful normal state of $N$ and $\alpha: N \rightarrow N \bar{\otimes} L^{\infty}(\mathbb{G})$ an w-preserving action of $\mathbb{G}$ on $N$. Then with $\tau$ being the scaling group of $\mathbb{G}$, we have

$$
\left(\sigma_{t}^{\omega} \otimes \tau_{-t}\right) \circ \alpha=\alpha \circ \sigma_{t}^{\omega} \quad(\forall t \in \mathbb{R}) .
$$

Proof. We fix some notation. Write $\sigma$ for $\sigma^{\omega}$. Let $S, R$ stand for the antipode and unitary antipode of $\mathbb{G}$, respectively. Denoting by $L^{2}(\mathbb{G})$ the standard representation Hilbert space of $L^{\infty}(\mathbb{G})$, we let $I, L$ be a conjugation and a strictly positive operator, respectively, over $L^{2}(\mathbb{G})$, satisfying $I L I=L^{-1}$ and $R(x)=I x^{*} I, \tau_{t}(x)=L^{i t} x L^{-i t}$ for every $x \in L^{\infty}(\mathbb{G}), t \in \mathbb{R}[23,24,38]$. Denote by $(\mathcal{H}, \mathrm{id}, \Gamma)$ the GNS construction for $(N, \omega)$. As discussed in $\S 1, \alpha$ is implemented by 
an isometric co-representation $U \in B(\mathcal{H}) \bar{\otimes} L^{\infty}(\mathbb{G})$ given by $((\mathrm{id} \otimes \theta)(U)) \Gamma(a)=\Gamma((\mathrm{id} \otimes \theta) \alpha(a))$ for all $\theta \in L^{1}(\mathbb{G}), a \in N$. The right leg of $U$ is thus characterized by

$$
\left(\omega_{\Gamma(a), \Gamma(b)} \otimes \mathrm{id}\right)(U)=(\omega \otimes \mathrm{id})\left(\left(b^{*} \otimes \mathbb{1}\right) \alpha(a)\right) \quad(\forall a, b \in N),
$$

and similarly

$$
\left(\omega_{\Gamma(a), \Gamma(b)} \otimes \mathrm{id}\right)\left(U^{*}\right)=(\omega \otimes \mathrm{id})\left(\alpha\left(b^{*}\right)(a \otimes \mathbb{1})\right) \quad(\forall a, b \in N) .
$$

Recall that as $\mathbb{G}$ is a LCQG, $U$ is in fact unitary [7, Corollary 4.12]. Kustermans showed in the proof of [22, Proposition 5.2] that, since $U$ is a unitary co-representation of $\mathbb{G}$ (i.e. $U \in$ $B(\mathcal{H}) \bar{\otimes} L^{\infty}(\mathbb{G})$ and $\left.(\mathrm{id} \otimes \Delta)(U)=U_{12} U_{13}\right)$, it satisfies $(\eta \otimes \mathrm{id})(U) \in D(S)$ and $S((\eta \otimes \mathrm{id})(U))=$ $(\eta \otimes \mathrm{id})\left(U^{*}\right)$ for every $\eta \in B(\mathcal{H})_{*}$. (The argument there uses $C^{*}$-algebra language, and treats co-representations in the sense that $(\Delta \otimes \mathrm{id})(U)=U_{13} U_{23}$, but the same reasoning works in our case.) For all $a, b \in N$, taking $\eta:=\omega_{\Gamma(a), \Gamma(b)}$ yields

$$
(\omega \otimes \mathrm{id})\left(\left(b^{*} \otimes \mathbb{1}\right) \alpha(a)\right) \in D(S) \quad \text { and } \quad S\left[(\omega \otimes \mathrm{id})\left(\left(b^{*} \otimes \mathbb{1}\right) \alpha(a)\right)\right]=(\omega \otimes \mathrm{id})\left(\alpha\left(b^{*}\right)(a \otimes \mathbb{1})\right)
$$

by (A.1) and (A.2). Since $S=R \circ \tau_{-i / 2}$ by definition (see [8] for this terminology), we have $(\omega \otimes \mathrm{id})\left(\left(b^{*} \otimes \mathbb{1}\right) \alpha(a)\right) \in D\left(\tau_{-i / 2}\right)$ and $\tau_{-i / 2}\left[(\omega \otimes \mathrm{id})\left(\left(b^{*} \otimes \mathbb{1}\right) \alpha(a)\right)\right]=R\left[(\omega \otimes \mathrm{id})\left(\alpha\left(b^{*}\right)(a \otimes \mathbb{1})\right)\right]$. By [8, Theorem 6.2], we get

$$
(\omega \otimes \mathrm{id})\left(\left(b^{*} \otimes \mathbb{1}\right) \alpha(a)\right) L^{-1 / 2} \subseteq L^{-1 / 2} I(\omega \otimes \mathrm{id})\left(\left(a^{*} \otimes \mathbb{1}\right) \alpha(b)\right) I,
$$

or equivalently, using that $I L I=L^{-1}$ and putting $K:=I L^{1 / 2}=L^{-1 / 2} I$,

$$
(\omega \otimes \mathrm{id})\left(\left(b^{*} \otimes \mathbb{1}\right) \alpha(a)\right) K \subseteq K(\omega \otimes \mathrm{id})\left(\left(a^{*} \otimes \mathbb{1}\right) \alpha(b)\right) .
$$

Taking adjoints gives

$$
(\omega \otimes \mathrm{id})\left(\alpha\left(b^{*}\right)(a \otimes \mathbb{1})\right) K^{*} \subseteq K^{*}(\omega \otimes \mathrm{id})\left(\alpha\left(a^{*}\right)(b \otimes \mathbb{1})\right) .
$$

The last two equations hold for every $a, b \in N$. If now $a \in D\left(\sigma_{-i}\right)$ and $b \in D\left(\sigma_{i}\right)$, then by the Tomita-Takesaki theory and (A.4),

$$
\begin{aligned}
(\omega \otimes \mathrm{id})\left(\left(b^{*} \otimes \mathbb{1}\right) \alpha(a)\right) K^{*} & =(\omega \otimes \mathrm{id})\left(\alpha(a)\left(\sigma_{i}(b)^{*} \otimes \mathbb{1}\right)\right) K^{*} \\
& \subseteq K^{*}(\omega \otimes \mathrm{id})\left(\alpha\left(\sigma_{i}(b)\right)\left(a^{*} \otimes \mathbb{1}\right)\right) \\
& \subseteq K^{*}(\omega \otimes \mathrm{id})\left(\left(\sigma_{-i}(a)^{*} \otimes \mathbb{1}\right) \alpha\left(\sigma_{i}(b)\right)\right) .
\end{aligned}
$$

Hence from (A.3),

$$
(\omega \otimes \mathrm{id})\left(\left(b^{*} \otimes \mathbb{1}\right) \alpha(a)\right) K^{*} K \subseteq K^{*} K(\omega \otimes \mathrm{id})\left(\left(\sigma_{i}(b)^{*} \otimes \mathbb{1}\right) \alpha\left(\sigma_{-i}(a)\right)\right) .
$$

Write $\nabla$ for the modular operator of $(N, \omega)$. Since $K^{*} K=L$, we conclude that

$$
\left(\omega_{\Gamma(a), \Gamma(b)} \otimes \mathrm{id}\right)(U) L \subseteq L\left(\omega_{\Gamma\left(\sigma_{-i}^{\omega}(a)\right), \Gamma\left(\sigma_{i}^{\omega}(b)\right)} \otimes \mathrm{id}\right)(U)=L\left(\omega_{\nabla \Gamma(a), \nabla^{-1} \Gamma(b)} \otimes \mathrm{id}\right)(U) .
$$


Since $\Gamma\left(D\left(\sigma_{-i}\right)\right)$ and $\Gamma\left(D\left(\sigma_{i}\right)\right)$ are cores of $\nabla$ and $\nabla^{-1}$, we infer that $\nabla \otimes L^{-1}$ commutes with $U$, namely $U\left(\nabla \otimes L^{-1}\right) \subseteq\left(\nabla \otimes L^{-1}\right) U$ (e.g. by [23, Lemma 5.9]). For every $x \in N$ we deduce that

$$
\begin{aligned}
\left(\sigma_{t} \otimes \tau_{-t}\right) \alpha(x) & =\left(\nabla^{i t} \otimes L^{-i t}\right) U(a \otimes \mathbb{1}) U^{*}\left(\nabla^{-i t} \otimes L^{i t}\right) \\
& =U\left(\nabla^{i t} \otimes L^{-i t}\right)(a \otimes \mathbb{1})\left(\nabla^{-i t} \otimes L^{i t}\right) U^{*} \\
& =U\left(\nabla^{i t} a \nabla^{-i t} \otimes \mathbb{1}\right) U^{*}=\alpha\left(\sigma_{t}(x)\right) .
\end{aligned}
$$

This completes the proof.

Acknowledgement. We are indebted to L. Zsidó for sending us the slides of his presentation [41]. We are also grateful to M. Alaghmandan, Y. Choi, M. Ghandehari, E. Samei, P. M. Sołtan, E. Spinu and V. G. Troitsky for fruitful conversations about the content of this paper, to P. R. Buckingham for many suggestions that led to an improved presentation of the material, and to the referee for his/her comments.

\section{REFERENCES}

1. B. Abadie and K. Dykema, Unique ergodicity of free shifts and some other automorphisms of $C^{*}$-algebras, J. Operator Theory 61 (2009), no. 2, 279-294. 1

2. T. Austin, T. Eisner, and T. Tao, Nonconventional ergodic averages and multiple recurrence for von Neumann dynamical systems, Pacific J. Math. 250 (2011), no. 1, 1-60. 1, 4

3. E. Bédos, G. J. Murphy, and L. Tuset, Co-amenability of compact quantum groups, J. Geom. Phys. 40 (2001), no. 2, 130-153. 10

4. E. Bédos and L. Tuset, Amenability and co-amenability for locally compact quantum groups, Internat. J. Math. 14 (2003), no. 8, 865-884. 3, 8, 10

5. C. Beyers, R. Duvenhage, and A. Ströh, The Szemerédi property in ergodic $W^{*}$-dynamical systems, J. Operator Theory 64 (2010), no. 1, 35-67. 1

6. F. P. Boca, Ergodic actions of compact matrix pseudogroups on $C^{*}$-algebras, Astérisque (1995), no. 232, 93-109, Recent advances in operator algebras (Orléans, 1992). 12

7. M. Brannan, M. Daws, and E. Samei, Completely bounded representations of convolution algebras of locally compact quantum groups, Münster J. Math., to appear, arXiv:1107.2094, v2. 9, 11,18

8. I. Ciorănescu and L. Zsidó, Analytic generators for one-parameter groups, Tôhoku Math. J. (2) 28 (1976), no. 3, 327-362. 15, 18

9. A. Connes, Classification of injective factors. Cases $I I_{1}, I I_{\infty}, I I I_{\lambda}, \lambda \neq 1$, Ann. of Math. (2) 104 (1976), no. 1, 73-115. 16

10. K. de Leeuw and I. Glicksberg, Applications of almost periodic compactifications, Acta Math. 105 (1961), 63-97. 1, 7, 8

11. P. Desmedt, J. Quaegebeur, and S. Vaes, Amenability and the bicrossed product construction, Illinois J. Math. 46 (2002), no. 4, 1259-1277. 3

12. N. Dunford and J. T. Schwartz, Linear operators. Part I. General theory, Wiley Classics Library, John Wiley \& Sons Inc., New York, 1988. 8 
13. R. Duvenhage, A mean ergodic theorem for actions of amenable quantum groups, Bull. Aust. Math. Soc. 78 (2008), no. 1, 87-95. 1, 2, 4, 6

14. _ Ergodicity and mixing of $W^{*}$-dynamical systems in terms of joinings, Illinois J. Math. 54 (2010), no. 2, 543-566. 1

15. _ Relatively independent joinings and subsystems of $\mathrm{W}^{*}$-dynamical systems, Studia Math. 209 (2012), no. 1, 21-41. 1

16. E. G. Effros and Z.-J. Ruan, Operator spaces, London Mathematical Society Monographs. New Series, vol. 23, Oxford University Press, 2000. 2, 4, 8

17. M. Enock, Sous-facteurs intermédiaires et groupes quantiques mesurés, J. Operator Theory 42 (1999), no. 2, 305-330. 17

18. M. Enock and J.-M. Schwartz, Algèbres de Kac moyennables, Pacific J. Math. 125 (1986), no. 2, 363-379. 3, 17

19. __ Kac algebras and duality of locally compact groups, Springer-Verlag, Berlin, 1992. 2

20. F. Fidaleo and F. Mukhamedov, Ergodic properties of Boguliubov automorphisms in free probability, Infin. Dimens. Anal. Quantum Probab. Relat. Top. 13 (2010), no. 3, 393-411. 1

21. K. Jacobs, Ergodentheorie und fastperiodische Funktionen auf Halbgruppen, Math. Z. 64 (1956), 298-338. 1, 7

22. J. Kustermans, Locally compact quantum groups in the universal setting, Internat. J. Math. 12 (2001), no. 3, 289-338. 18

23. J. Kustermans and S. Vaes, Locally compact quantum groups, Ann. Sci. École Norm. Sup. (4) 33 (2000), no. 6, 837-934. 2, 14, 17, 19

24. Locally compact quantum groups in the von Neumann algebraic setting, Math. Scand. 92 (2003), no. 1, 68-92. 2, 17

25. A. Maes and A. Van Daele, Notes on compact quantum groups, Nieuw Arch. Wisk. (4) 16 (1998), no. 1-2, 73-112. 2, 5, 10, 12, 13, 15

26. T. Masuda, Y. Nakagami, and S. L. Woronowicz, $A C^{*}$-algebraic framework for quantum groups, Internat. J. Math. 14 (2003), no. 9, 903-1001. 3

27. C. P. Niculescu, A. Ströh, and L. Zsidó, Noncommutative extensions of classical and multiple recurrence theorems, J. Operator Theory 50 (2003), no. 1, 3-52. 1, 4, 6, 7, 8, 11

28. V. Runde, Lectures on amenability, Lecture Notes in Mathematics, vol. 1774, Springer-Verlag, Berlin, 2002. 16

29. __ Completely almost periodic functionals, Arch. Math. (Basel) 97 (2011), no. 4, 325331. 8

30. P. M. Sołtan, Quantum Bohr compactification, Illinois J. Math. 49 (2005), no. 4, 1245-1270. 9

31. Ş. Strătilă, Modular theory in operator algebras, Abacus Press, Tunbridge Wells, England, 1981. 2, 17

32. M. Takesaki, Tomita's theory of modular Hilbert algebras and its applications, Lecture Notes in Mathematics, Vol. 128, Springer-Verlag, Berlin, 1970. 2

33. _ Conditional expectations in von Neumann algebras, J. Funct. Anal. 9 (1972), 306321. 17 
34. , Theory of operator algebras. II, Encyclopaedia of Mathematical Sciences, vol. 125, Springer-Verlag, Berlin, 2003. 2, 3, 17

35. R. Tomatsu, Amenable discrete quantum groups, J. Math. Soc. Japan 58 (2006), no. 4, 949964. 3

36. S. Vaes, The unitary implementation of a locally compact quantum group action, J. Funct. Anal. 180 (2001), no. 2, 426-480. 17

37. S. Vaes and L. Vainerman, On low-dimensional locally compact quantum groups, Locally compact quantum groups and groupoids (Strasbourg, 2002), IRMA Lect. Math. Theor. Phys., vol. 2, de Gruyter, Berlin, 2003, pp. 127-187. 17

38. A. Van Daele, Locally compact quantum groups. A von neumann algebra approach, preprint, arXiv:0602212, v1, 2006. 2, 17

39. S. L. Woronowicz, A remark on compact matrix quantum groups, Lett. Math. Phys. 21 (1991), no. $1,35-39.9,10$

40. _ Compact quantum groups, Symétries quantiques (Les Houches, 1995), NorthHolland, Amsterdam, 1998, pp. 845-884. 2, 5, 10, 12, 13, 14

41. L. Zsidó, Splitting noncommutative dynamical systems in almost periodic and weakly mixing parts, presented in "International Conference on Operator Theory", Timişoara, Romania, 2012. $1,7,8,11,19$

Department of Mathematical and Statistical Sciences, University of Alberta, Edmonton, Alberta T6G 2G1, CANADA

E-mail address: vrunde@ualberta.ca

Department of Mathematical and Statistical Sciences, University of Alberta, Edmonton, Alberta T6G 2G1, CANADA

E-mail address: viselter@ualberta.ca 